\title{
Dynamics and stability of radiation-driven double ablation front structures
}

\author{
V. Drean, ${ }^{1, a)}$ M. Olazabal-Loumé, ${ }^{1}$ J. Sanz, ${ }^{2}$ and V. T. Tikhonchuk ${ }^{1}$ \\ ${ }^{1}$ CELIA, Université Bordeaux 1-CNRS-CEA, 351 cours de la Libération, 33405 Talence, France \\ ${ }^{2}$ ETSI Aeronauticos, Universidad Politecnica de Madrid, Madrid 28040, Spain
}

(Received 3 September 2010; accepted 12 October 2010; published online 1 December 2010)

\begin{abstract}
The dynamics of double ablation front (DAF) structures is studied for planar targets with moderate atomic number ablators. These structures are obtained in hydrodynamic simulations for various materials and laser intensities and are qualitatively characterized during the acceleration stage of the target. The importance of the radiative transport for the DAF dynamics is then demonstrated. Simulated hydrodynamic profiles are compared with a theoretical model, showing the consistency of the model and the relevant parameters for the dynamics description. The stability of DAF structures with respect to two-dimensional perturbations is studied using two different approaches: one considers the assumptions of the theoretical model and the other one a more complete physics. The numerical simulations performed with both approaches demonstrate good agreement of dispersion curves. (C) 2010 American Institute of Physics. [doi:10.1063/1.3509108]
\end{abstract}

\section{INTRODUCTION}

In inertial confinement fusion (ICF), a shell of solid deuterium-tritium mixture is accelerated to high velocities by direct laser or by $\mathrm{x}$-ray irradiation of the target surface. The imploding shell acts as a piston, raising the pressure of the enclosed deuterium-tritium (DT) gas. The shell slows down as the central pressure builds up, until it comes to a full stop. At this point, the central spot is dense and hot enough to trigger the onset of the ignition process.

Most high-gain target designs feature a capsule with a thick (hundreds of microns) DT ice layer inside a very thin shell (a few microns in thickness) currently made of light materials with low atomic number $(Z)$, such as plastic or glass. Since the thin overcoat is quickly ablated off in the early stages of the driving laser pulse, the main ablator is hydrogenic, and the DT ice plays both roles of an ablator material and a thermonuclear fuel. However, moderate $Z$ ablators may present some advantages.

Two limits of ablation process are known. ${ }^{1-9}$ One corresponds to the ablation driven by $\mathrm{x}$-ray radiation (indirect drive); another corresponds to the direct laser ablation of low- $Z$ materials. In the former case, the radiation is absorbed directly in a solid matter, and the temperature of ablated vapors (plasma) is equal to the effective temperature of radiation. In the latter case, the optical radiation is absorbed in low density vapors, where the density is approximately equal to the critical density, depending on the radiation wavelength. The energy from the absorption zone to the ablation layer is transported by electrons.

In both limits, the ablation process is described by a rarefaction wave, where the density and temperature of plasma are smooth, monotonic functions of the distance and time. However, in the case of moderate $Z$ materials, the ablation front demonstrates a more complicated double-layer structure. Numerical simulations ${ }^{10-13}$ and experiments ${ }^{14}$

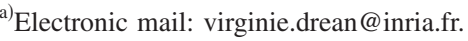

demonstrate that radiative and electron transports are both important in this case.

Recent cryogenic implosion experiments on OMEGA laser ${ }^{15-17}$ have suggested that double-layer structures may suppress the fuel preheating. Other experiments on GEKKOXII laser ${ }^{18}$ demonstrated that such structures can improve the ablation front stability with respect to surface perturbations, but no qualitative studies of these structures have been carried out yet.

In this work, double ablation front (DAF) structures are studied in planar targets made of moderate $Z$ ablators, such as aluminum, silica, plastic, and brominated plastic. First, the DAF structures are obtained in simulations with the hydrodynamic radiative code CHIC (Refs. 19-25) and are qualitatively characterized during the acceleration stage of the target.

They are then compared with predictions of a simplified physical model ${ }^{16}$ developed recently. The comparison permits us to validate the model and to define the processes responsible for the formation of a DAF.

In the second part of this work, the stability of DAF structures with respect to two-dimensional (2D) perturbations is studied with the code PERLE. ${ }^{26,27}$ This code is dedicated to the linear stability study of unsteady hydrodynamic flows and was modified to take into account the radiative transfer in a diffusion approximation.

The 2D simulations made with the code CHIC including a more complete physics have confirmed the results obtained with the code PERLE. The dispersion curves obtained for moderate $Z$ ablators demonstrate a partial suppression of the ablative Rayleigh-Taylor instability (RTI), opening thus the possibility of optimization of ICF target designs.

\section{NUMERICAL SIMULATIONS OF THE DAF STRUCTURES}

One-dimensional (1D) radiation hydrodynamic codes are intensively used for target design in ICF. In our studies, we use a Lagrangian code CHIC, ${ }^{19-25}$ developed in CELIA, and 


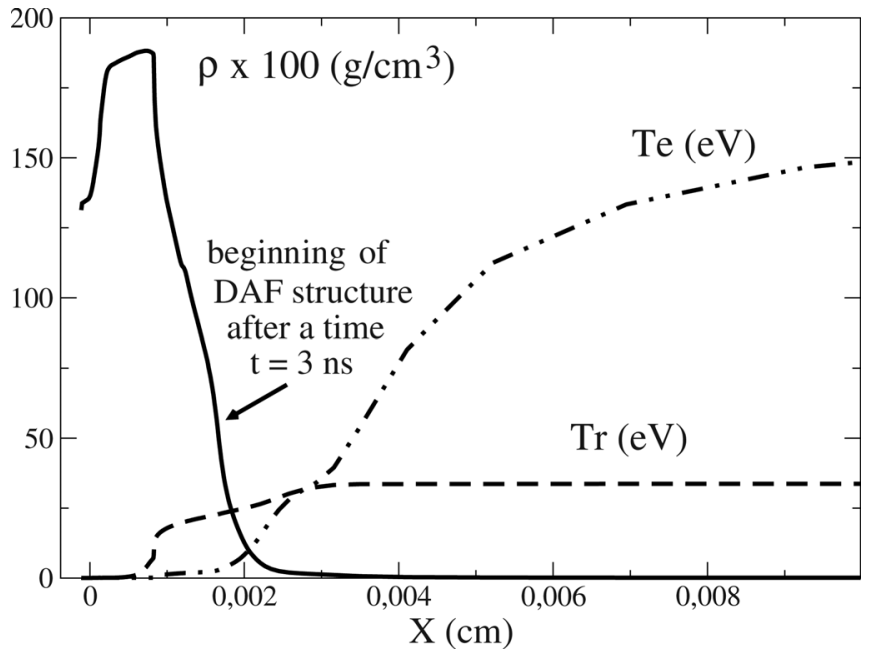

FIG. 1. Density, electron, and radiative temperature profiles for the aluminum target for $I_{L}=1 \mathrm{TW} / \mathrm{cm}^{2}$ at $t=3 \mathrm{~ns}$.

dedicated to ICF calculations. It operates in $1 \mathrm{D}$ and $2 \mathrm{D}$ axially symmetric geometries and includes three-dimensional (3D) ray tracing for laser beam propagation. The laser energy release is modeled by inverse bremsstrahlung absorption; the electron thermal transport can be described in the classical Spitzer-Harm ${ }^{28}$ approximation, with flux limitation, or with a nonlocal transport model. The equation of state is based on SESAME tables and QEOS tables, and on the ThomasFermi model for ionization. A multigroup diffusion approximation of the radiation transport is included into the code. The radiative temperature $T_{r}=\left(U_{r} / a\right)^{1 / 4}$ is calculated directly from the radiative energy $U_{r}$ in each mesh point and at each iteration in time, where $a=4 \sigma_{B} / c$ is defined by the StefanBoltzmann constant $\sigma_{B}$ and the light velocity $c$. In this work, the magnetic field generation and the nonlocal conduction have not been used.

We consider planar foils of the thickness of $20-30 \mu \mathrm{m}$, irradiated by a laser pulse of a constant intensity. The pulse is turned on instantaneously or with a linear ramp during a hundred of picoseconds. The pulse duration is about 3-5 ns for intensities ranging from one to hundreds of $\mathrm{TW} / \mathrm{cm}^{2}$. These parameters correspond to a typical laser pulse for the ICF target compression.
The DAF structures are identified by two ablation fronts, separated by an almost constant density region called "plateau." According to Refs. 16-18, these fronts are driven by different energy transport mechanisms: the dense front (called the main front), near the cold material is dominated by the radiative transport, while the front near the corona region (called the second front) is supported by both the electron and radiative transports.

\section{A. DAF characterization}

The DAF structures are studied in the range of laser light intensities $I_{L}$ of $1-500 \mathrm{TW} / \mathrm{cm}^{2}$. As reference materials, we consider $\mathrm{CHBr}_{4 \%}, \mathrm{Al}$, and $\mathrm{SiO}_{2}$ with initial densities of 1.45 , 2.7 , and $2.2 \mathrm{~g} / \mathrm{cm}^{3}$, respectively.

\section{DAF structures in Al targets}

The radiative effects are enhanced in $\mathrm{Al}$ targets and provide clear signatures of DAF structures. For lowest intensities, in the range of $I_{L}=1-50 \mathrm{TW} / \mathrm{cm}^{2}$, two fronts separated by a density plateau are formed after a time $t>2 \mathrm{~ns}$. Before the shock break out at the rear side of the foil, no clear DAF is visible on hydrodynamic profiles. For $I_{L}=1 \mathrm{TW} / \mathrm{cm}^{2}$, the DAF structure appears only after $t=3 \mathrm{~ns}$ (Fig. 1). This delay is rather long because the radiative effects are weak at low laser intensities and they induce only small modifications of hydrodynamic profiles.

In Fig. 1, the target is irradiated from the right. A thermal wave is created and propagates into the matter, which is then heated as it is seen on the electron temperature profile $T_{e}$. As the temperature decreases, a shock is launched at the foot of the thermal wave. It is indicated by a local increase of the density. The radiative temperature $T_{r}$ is almost constant in the hot region, and decreases near the foot of the heat wave, where the matter is colder, denser, and then more opaque. The $\mathrm{x}$ rays are emitted in the corona and absorbed in colder regions, where they are converted and re-emitted. A second foot in the thermal wave is then created, leading to a double ablation front structure.

For higher intensities $\left(I_{L}=10-50 \mathrm{TW} / \mathrm{cm}^{2}\right)$, the DAF structure is created before the shock break out at the rear side of the foil. The position of the second front is at the point where the temperatures $T_{e}$ and $T_{r}$ are equal (Fig. 2). They are
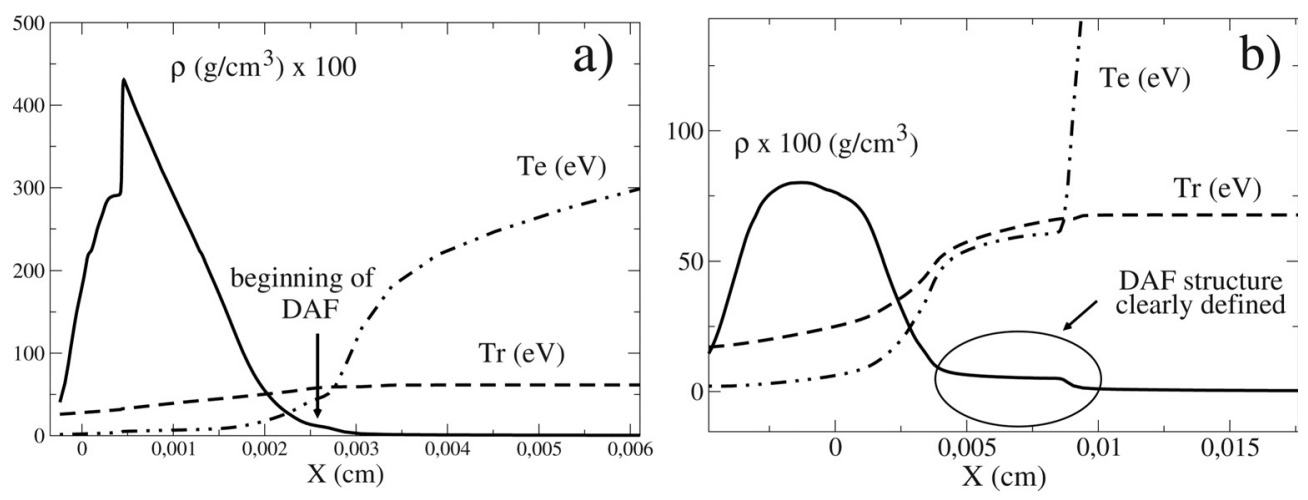

FIG. 2. Density, electron, and radiative temperature profiles for $I_{L}=10 \mathrm{TW} / \mathrm{cm}^{2}$ at time $t=0.9 \mathrm{~ns}$ (a) before acceleration stage and at $3 \mathrm{~ns}$ (b) during the acceleration stage for the aluminum target. 

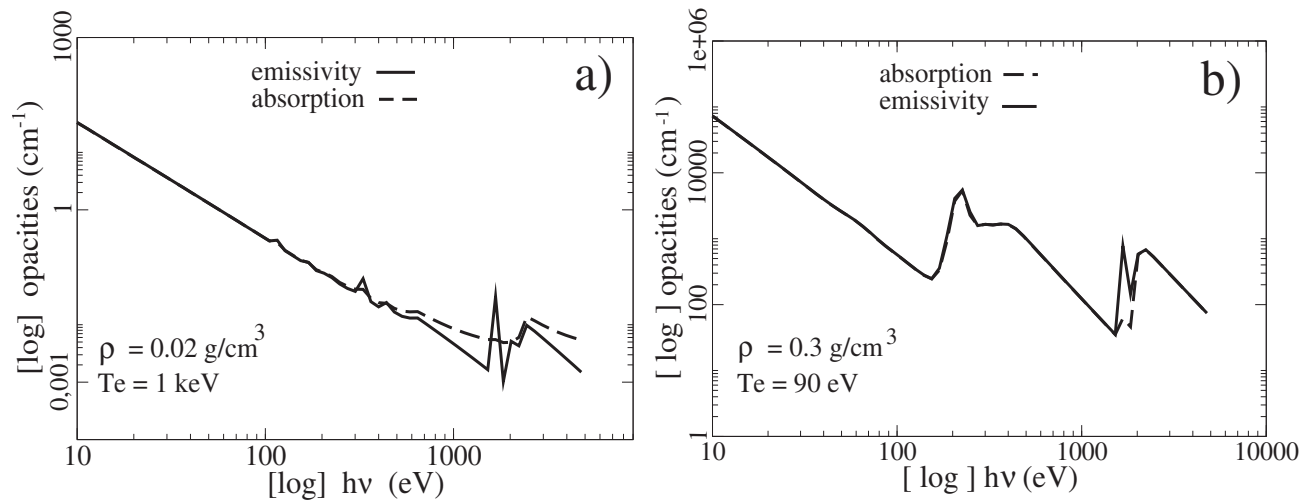

FIG. 3. Emissivity and absorption for aluminum target in the corona (a) and in the ablation region (b).

almost equal in the whole plateau region. The plateau is well defined only during the acceleration stage, after the shock break out.

In the case of low intensities $\left(1-10 \mathrm{TW} / \mathrm{cm}^{2}\right)$ with a linear ramp $(\sim 100 \mathrm{ps})$, the radiative transport is not sufficient for the DAF creation during the simulation time $(\sim 3 \mathrm{~ns}$ for the $20 \mu \mathrm{m}$ target).

For higher intensities $\left(I_{L}=100-300 \mathrm{TW} / \mathrm{cm}^{2}\right)$, the DAF structures are very well defined, independently of the ramp duration, before and during the acceleration stage. Strong radiative effects are clearly visible on hydrodynamic profiles: the energetic photons are emitted from the corona region (high temperatures and low densities), from the $K$ - and $L$-shell transitions of $\mathrm{Al}$ atoms. These photons are absorbed in more opaque regions, at the thermal wave foot, where the temperature is lower and the density is higher, creating then a second ablation front. This can be seen from the plots of emissivity and absorption shown in Fig. 3 for aluminum in the ablation and corona regions. Typical values of density and electron temperature in these regions are $0.02 \mathrm{~g} / \mathrm{cm}^{3}$ and $1 \mathrm{keV}$ for the corona, and $0.3 \mathrm{~g} / \mathrm{cm}^{3}$ and $90 \mathrm{eV}$ near the plateau, respectively.

In the corona region [panel (a)], the $K$-shell transitions are visible for photon energies in the range of $\sim 2-3 \mathrm{keV}$, and $L$-shell transitions in the range of $\sim 200-300 \mathrm{eV}$. In the ablation region [panel (b)], the photons are reabsorbed (absorption and emissivity are almost equal) and are re-emitted with the temperature of $\sim 1.5 \mathrm{keV}$. These photons are reabsorbed in a more opaque region, creating the main ablation front (see Fig. 4).

For higher intensities in the range of $I_{L}$ $=400-500 \mathrm{TW} / \mathrm{cm}^{2}$, the DAF structures exist from the first moments of irradiation: at $t=0.2 \mathrm{~ns}$, the second front is already created. However, as we consider relatively thin planar foils, the maximum density at the main ablation front decreases and becomes very low $\left(\rho \ll \rho_{0}\right)$ after the first $2-3$ ns of interaction ( $t>1.5 \mathrm{~ns}$ typically). The matter is quickly ablated because of the enhanced radiative effects before the acceleration sets in. For longer times around $3 \mathrm{~ns}$, the second front can be as dense as the main one, and the temperature is almost the same for both fronts. The second front can then be as opaque as the main one and can absorb more incoming photons: the DAF structure is modified before it inverses itself (Fig. 5). The time interval needed for DAF structure creation in $\mathrm{Al}$ is presented in Fig. 6.

The intensity needed to create such structures at the time $t$ scales as $t^{-2.3}$. For higher laser intensities, the radiative effects are stronger and the DAF structures appear early. The dynamics of the DAF structures is then dependent on the laser intensity, as seen in Fig. 7.
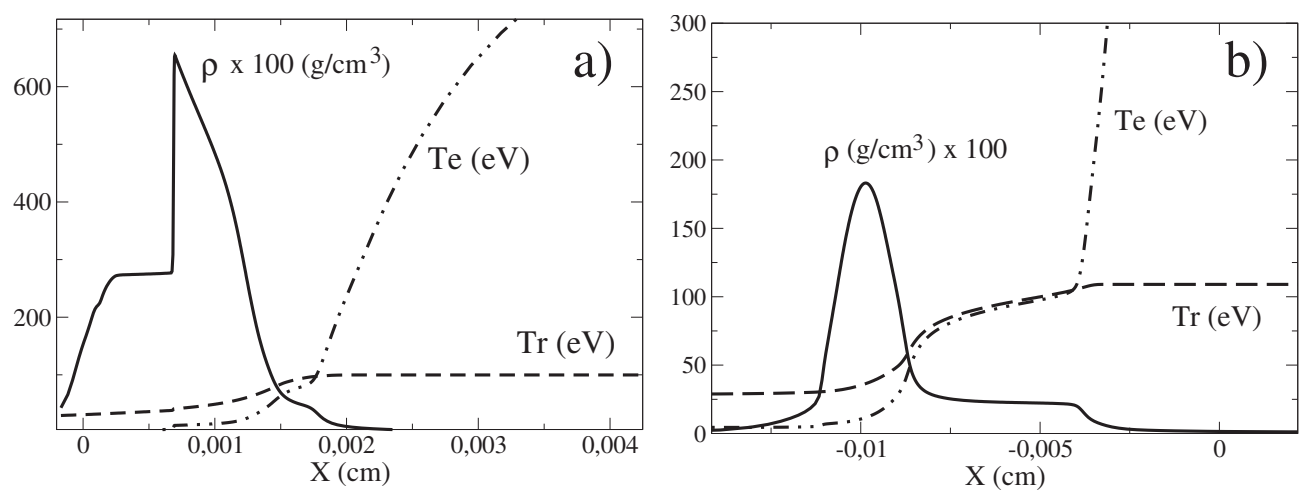

FIG. 4. Density, electron, and radiative temperature profiles for $I_{L}=100 \mathrm{TW} / \mathrm{cm}^{2}$ for the aluminum target at $0.4 \mathrm{~ns}$ (a) (before the acceleration stage) and at $3 \mathrm{~ns}$ (b) (during the acceleration stage). 

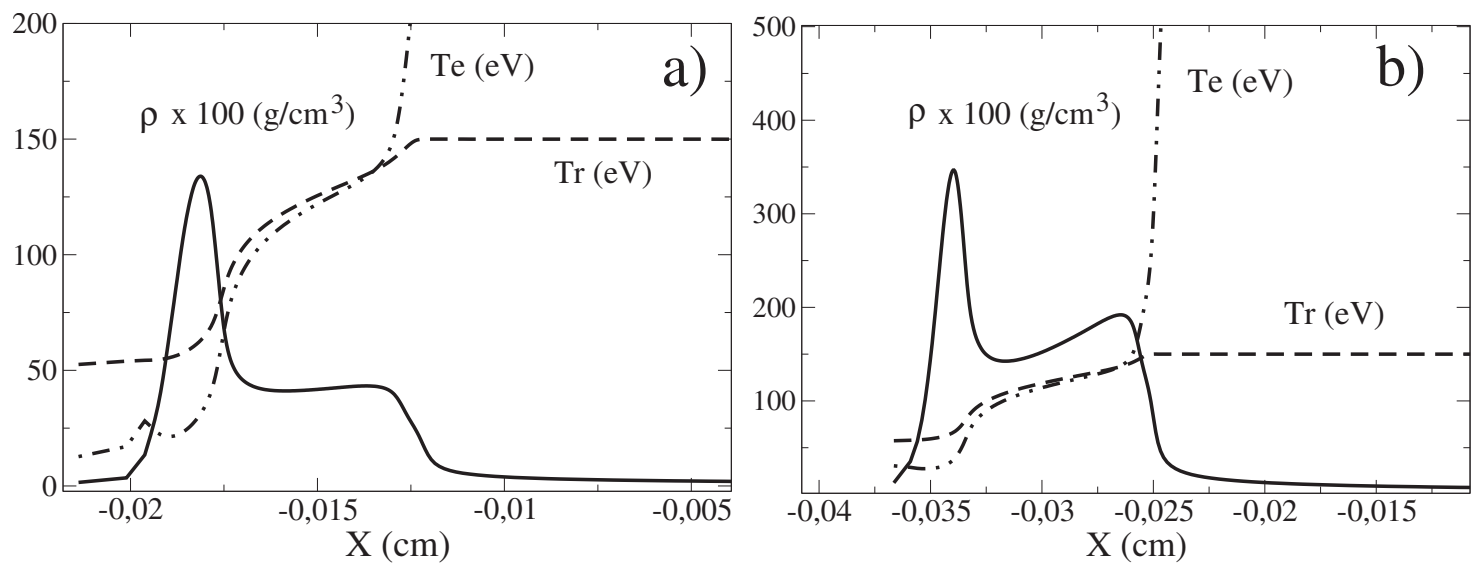

FIG. 5. Density, electron, and radiative temperature profiles for $I_{L}=400 \mathrm{TW} / \mathrm{cm}^{2}$ at time $2.2 \mathrm{~ns}$ (a) and $3 \mathrm{~ns}$ (b) for the aluminum target.

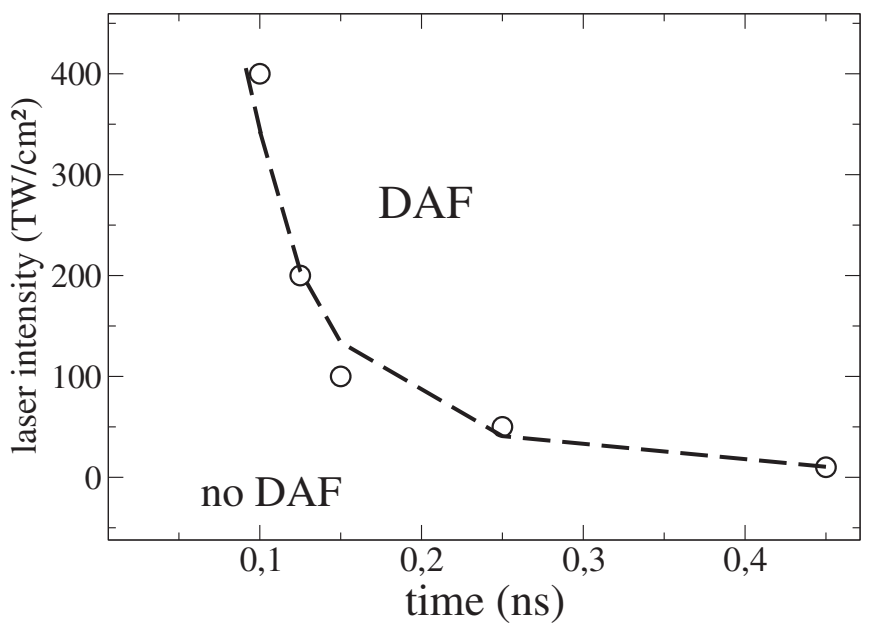

FIG. 6. Domain where the DAF structures exist as a function of time for the aluminum foil of $20 \mu \mathrm{m}$ thick.

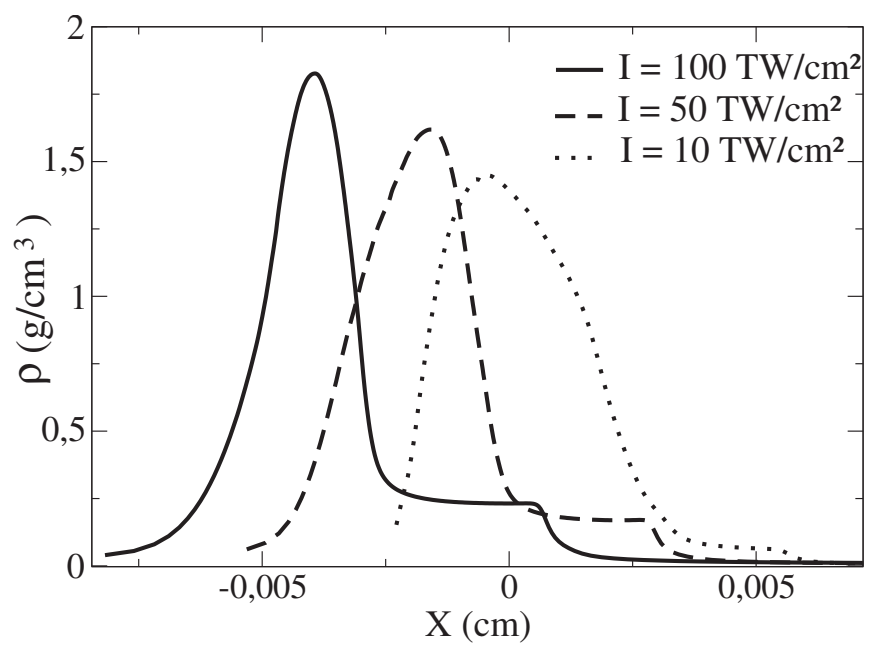

FIG. 7. Density profiles during acceleration stage for three laser intensities at $2 \mathrm{~ns}$ for the aluminum target.

\section{DAF structures in $\mathrm{CHBr}$ targets}

The doping of $\mathrm{Br}$ in plastic targets allows to control the amount of radiation emission and thus to see its effect on the DAF. We studied $\mathrm{CH}$ targets with three doping levels of $\mathrm{Br}: \mathrm{CHBr}_{4}\left(\rho_{0}=1.45 \mathrm{~g} \mathrm{~cm}^{-3}\right)$, with $4.2 \%$ doping, as $\mathrm{C}_{50} \mathrm{H}_{45.8} \mathrm{Br}_{4.2}, \mathrm{CHBr}_{3} \quad\left(\rho_{0}=1.35 \mathrm{~g} \mathrm{~cm}^{-3}\right)$, with $3.3 \%$, as $\mathrm{C}_{50} \mathrm{H}_{41.5} \mathrm{Br}_{3.3}$, and $\mathrm{CHBr}\left(\rho_{0}=1.26 \mathrm{~g} \mathrm{~cm}^{-3}\right)$, with $2 \%$, as $\mathrm{C}_{50} \mathrm{H}_{48} \mathrm{Br}_{2}$.

A $20 \mu \mathrm{m}$ thick target is irradiated at a constant intensity of $200 \mathrm{TW} / \mathrm{cm}^{2}$ with a $500 \mathrm{ps}$ linear ramp. This set of parameters corresponds to the experimental data. ${ }^{18}$ The calculated density and the temperature profiles are shown in Fig. 8.

The amount of doping influences the DAF structures shape and their spatial positions. The radiative temperature is higher for $\mathrm{CHBr}_{4}$ and the DAF structure is better defined. In the $\mathrm{CH}$ target, the laser energy conversion into radiation is much less efficient and only one ablation front can be created

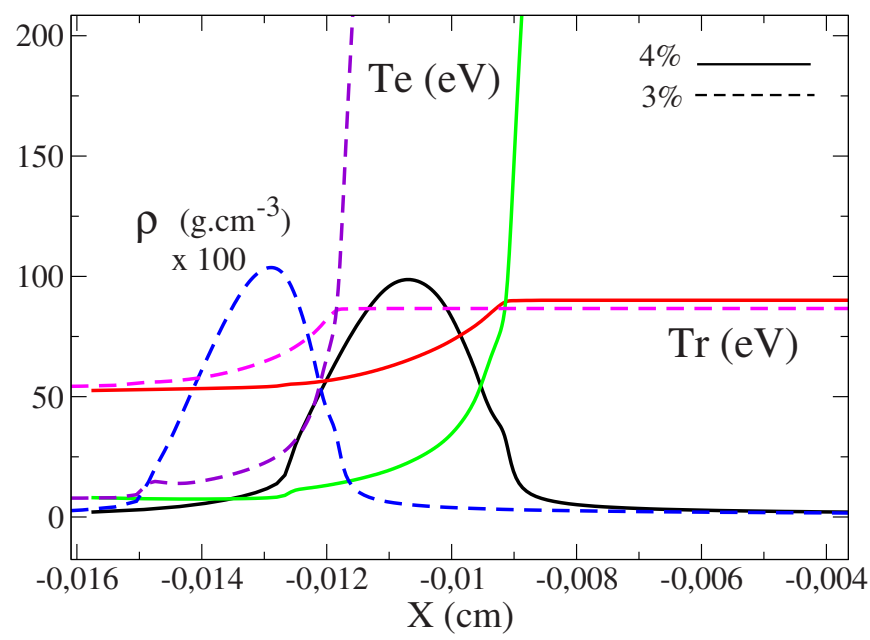

FIG. 8. (Color online) Density, electron, and radiative temperature profiles for the $4 \%$ and $3 \% \mathrm{CHBr}$ targets for $I_{L}=100 \mathrm{TW} / \mathrm{cm}^{2}$ at $2.2 \mathrm{~ns}$. 


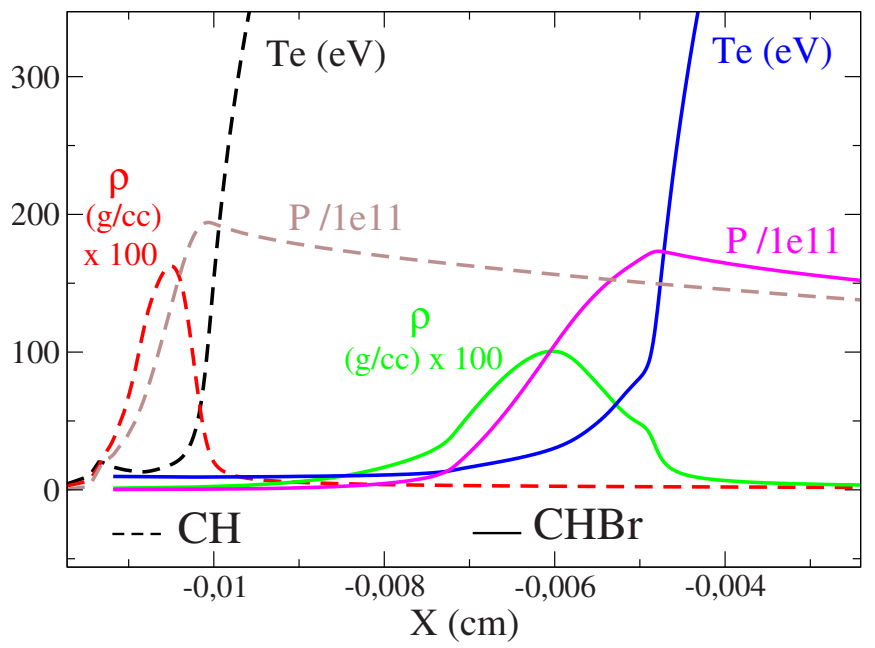

FIG. 9. (Color online) Density and temperature profiles for $\mathrm{CH}$ and $\mathrm{CHBr}_{4 \%}$ at $t=2.2 \mathrm{~ns}$.

(see Fig. 9). The profiles obtained for the $\mathrm{CH}$ target are in good agreement with the results presented in Ref. 18. However, this is not the case for the $\mathrm{CHBr}$ target. The differences are likely due to the very low photon cutoff energy in the radiative transport model of Ref. 18. This will be further discussed in Sec. III B.

The DAF structures have been obtained for other materials, such as $\mathrm{SiO}_{2}$ and $\mathrm{CHBr}$. In accordance with Refs. 10 and 11, the DAF exists for materials with $Z>6$ (carbon), where the radiation transport is efficient. Consequently, the DAF was not observed in $\mathrm{CH}(Z=3.5)$, but it has a strong effect on hydrodynamics in $\mathrm{SiO}_{2}(Z=10)$ and $\mathrm{Al}(Z=13)$. In $\mathrm{CHBr}$ targets $(Z \sim 5)$, the radiative effects depend on the concentration of $\mathrm{Br}$, and the DAF structure is not well defined compared to $\mathrm{Al}$ or $\mathrm{SiO}_{2}$.

The structures obtained in silica are very similar to the ones obtained in aluminum (Fig. 10). The intensity threshold is almost the same, $\sim 20 \mathrm{TW} / \mathrm{cm}^{2}$.

\section{Influence of the ionization model and the equation of state on the DAF}

In order to develop an analytical theory for DAF structure formation and stability, it is instructive to isolate the dominant physical effects and to define a simpler model. To investigate these effects, we artificially varied the ionization level in our simulations and used an ideal gas equation of state.

Then we compared CHIC simulations with two ionization models: the standard Thomas-Fermi (TF) model with the ionization level dependent on the temperature, and a much simpler model that assumes a totally ionized (TI) plasma. The DAF structures obtained in both simulations are similar, with small quantitative differences:

(a) the maximum density at the main front is a little lower for the TI calculation $\left(\rho_{\max } \sim 2.93 \mathrm{~g} \mathrm{~cm}^{-3}\right.$ for TI model, while $\rho_{\max } \sim 3.03 \mathrm{~g} \mathrm{~cm}^{-3}$ for TF model);

(b) the radiative temperature is a little bit higher before the ablation region $\left(T_{r} \sim 106 \mathrm{eV}\right.$ for TI model, while $T_{r}$ $\sim 100 \mathrm{eV}$ for $\mathrm{TF}$ model), but $T_{e}$ and $T_{r}$ are almost equal in the plateau region;

(c) the density plateau is longer for TI calculation ( $\sim 4 \mu \mathrm{m}$ more than TF model);

(d) the characteristic front thickness is smaller for the TI calculation; and

(e) the shock break out time is almost the same ( $t=0.7 \mathrm{~ns})$ for both calculations, so the temporal evolution of DAF is the same.

The ionization model influences the laser energy absorption, thermal conductivity, and specific heat because the absorption coefficient depends on the ionization level, which is larger for TI calculation (the maximum charge state is reached in the corona region, but not in the ablation front, where the temperature is low). These variations explain a larger width of the plateau, as well as the differences in density and temperature levels.

The same observations were drawn from the simulations with an ideal gas equation of state: the DAF structures are very similar. Thus, simplified assumptions concerning a fixed ionization level and an ideal gas equation of state are sufficient to describe the DAF structures and are relevant to study their dynamics and stability.

\section{TEMPORAL EVOLUTION OF THE DAF STRUCTURES}

The DAF structures can be described in terms of the width of the plateau and the characteristic lengths at the den-
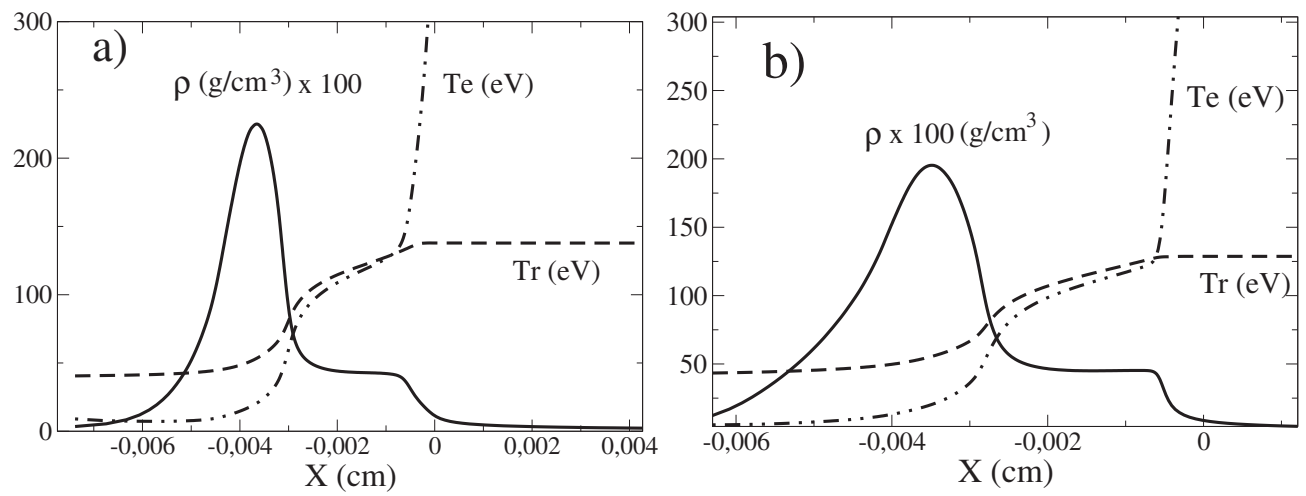

FIG. 10. Density, electron and radiative temperatures profiles for $I_{L}=100 \mathrm{TW} / \mathrm{cm}^{2}$ at time $1.2 \mathrm{~ns}$ for the aluminum (a) and silica (b) targets. 

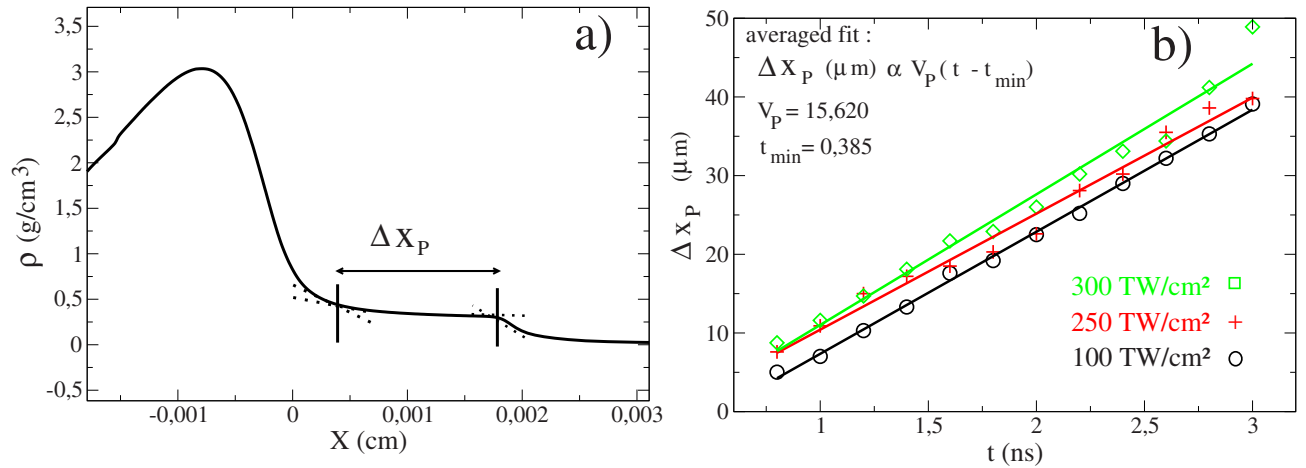

FIG. 11. (Color online) Definition of the plateau size $\Delta x_{P}(\mu \mathrm{m})$ (a); evolution of $\Delta x_{P}$ in time $t$ for different laser intensities in the aluminum target (b).

sity jumps. The temporal evolution of these parameters is described below.

\section{A. Temporal evolution of the plateau}

We define the plateau width $\Delta x_{P}$ by choosing two characteristic points on the density profiles, as shown in Fig. 11. The distance $\Delta x_{P}$ for a given laser intensity increases with time (Figs. 11 and 12). This is due to the fact that the characteristic flow velocities are different for each ablation front. The main front has a greater velocity than the second one (Fig. 13). The plateau density $\left(\rho \sim 0.3 \mathrm{~g} / \mathrm{cm}^{3}\right)$ does not vary in time, as well as the electron and radiation temperatures. In the hot corona region, $T_{r}$ is almost constant, and its value slowly varies in time.

The difference in the front velocities and the plateau expansion are explained by different opacities of the fronts: the main front is more opaque so its velocity is higher than for the second. Photons emitted from the hot corona are absorbed in the main front and increase the energy and the velocity locally.

The dependence of $\Delta x_{P}$ on time is almost linear (Fig. 11) and it can be approximated by

$$
\Delta x_{P}=V_{P}\left(t-t_{\min }\right) .
$$

The parameter $t_{\min }$ is the time for the DAF structure to appear. For laser intensities in the range of $100-300 \mathrm{TW} / \mathrm{cm}^{2}$, the evolution of the plateau width is similar. We found that $V_{P} \sim 15 \mu \mathrm{m} / \mathrm{ns}$ and $t_{\min }=0.38 \mathrm{~ns}$ for the runs where the in- tensity is constant in time, and $t_{\min }=0.7 \mathrm{~ns}$ for the runs where intensity has an initial ramp of 100 ps. Thus, the ramp affects $t_{\min }$ but has a very weak effect on $V_{P}$. For higher $Z$ materials, the silica, for example, the plateau expansion velocity is higher, $V_{P}=25.6 \mu \mathrm{m} / \mathrm{ns}$, and it appears earlier, $t_{\min }=0.2 \mathrm{~ns}$.

\section{B. Dependence of the plateau parameters on model assumptions}

We studied the influence of the laser intensity profile, the electron flux limitation, and the radiation flux parameters on the DAF structure. Two laser intensity shapes are considered: a constant intensity $I_{L}=500 \mathrm{TW} / \mathrm{cm}^{2}$ and a $1 \mathrm{~ns}$ linear ramp followed by the same constant intensity (see Fig. 13). The flux limiter is $f=0.09$.

For the laser pulse with a linear ramp, $\Delta x_{P}$ is smaller, as seen in Fig. 14, and the DAF structure is not as well defined as for the constant intensity pulse. Less energy is then released in the first moments of the laser irradiation and the time $t_{\min }$ for the DAF structure to appear is then longer. As this ramp affects very little the parameter $V_{P}$, the plateau width $\Delta x_{P}$ decreases.

For the run where the flux is limited, we have then a smaller expansion velocity because of the stronger flux limitation, but the parameter $t_{\min }$ practically did not vary. We found that $V_{P}=12.5 \mu \mathrm{m} / \mathrm{ns}$ and $t_{\min }=0.54 \mathrm{~ns}$. Thus, for a stronger flux limitation, the plateau expansion velocity decreases.
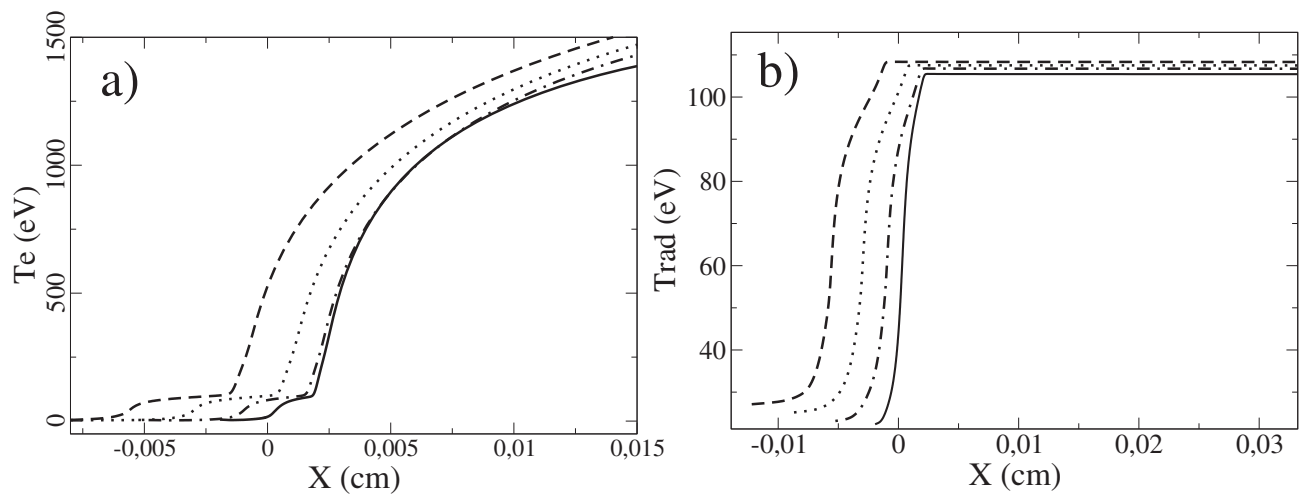

FIG. 12. Temperature profiles $T_{e}$ (a) and $T_{r}$ (b) for $I_{L}=100 \mathrm{TW} / \mathrm{cm}^{2}$ at four time moments during acceleration stage of Al target: $t=1(-), 2(\cdot-), 3(. \cdot)$, and $3.5 \mathrm{~ns}(-)$. 

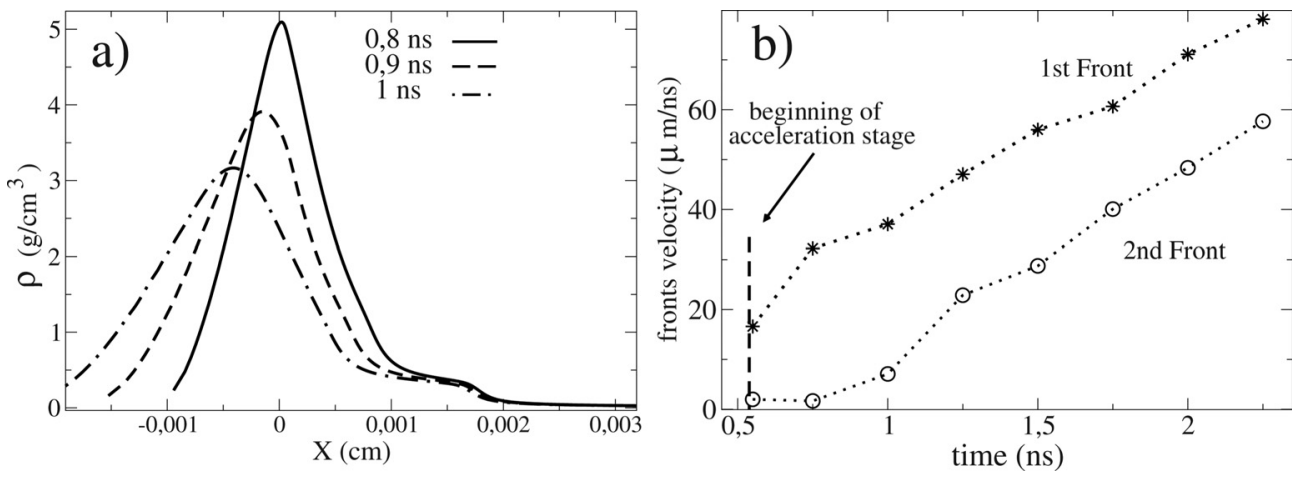

FIG. 13. Evolution of DAF density profiles (a) and front velocities (b) for the $\mathrm{SiO}_{2}$ target.

The radiation transport in simulations is characterized by two parameters: the number of energy groups and the maximum energy $E_{\max }$. We considered four cases: (a) 100 groups and $E_{\max }=70 \mathrm{keV}$ (baseline set); (b) 10 groups, $E_{\max }$ $=70 \mathrm{keV}$; (c) 100 groups, $E_{\max }=1 \mathrm{keV}$; and (d) 100 groups, $E_{\max }=100 \mathrm{keV}$.

We choose these different parameters in order to define the maximum energy $E_{\max }$ necessary to describe the main physical processes induced by high energies related to the photon transport, and how many energy groups of photons are needed to provide a sufficient resolution in simulation. Thus, with a high number of energy groups and a high maximum energy, we are sure that every photon energy is considered.

If the cutoff energy is high enough, the number of groups has a little effect on $\Delta x_{P}$, as almost all photon energies are taken into account for $E_{\max }=100 \mathrm{keV}$. As well as for $E_{\max }=70 \mathrm{keV}$, we found practically no difference for the evolution in time of $\Delta x_{P}$. Thus, the photons responsible for the DAF structure have energies significantly lower than 70 $\mathrm{keV}$.

On the contrary, in the case of very small $E_{\max }=1 \mathrm{keV}$, the radiative effects are underestimated; the energy cutoff is then too small. We conclude that photon energies above 1 $\mathrm{keV}$ are responsible for the DAF formation. It can be easily

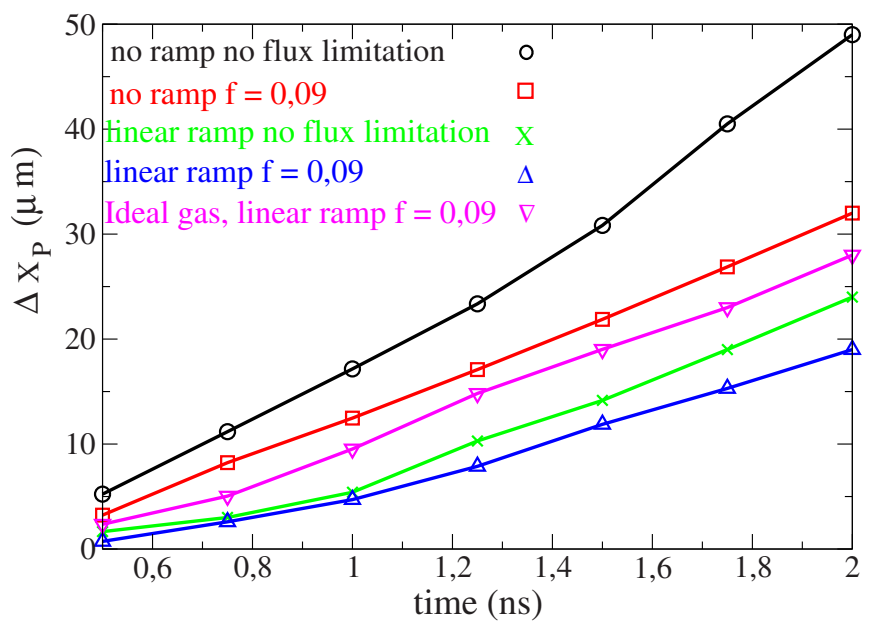

FIG. 14. (Color online) Influence of laser intensity shape and the electron flux limitation on $\Delta x_{P}(\mu \mathrm{m})$ for the $\mathrm{SiO}_{2}$ target at $I_{L}=500 \mathrm{TW} / \mathrm{cm}^{2}$. understood as the photons with energies of $\sim 6 T_{r}$ dominate the radiative transport. For the $\mathrm{SiO}_{2}$ target irradiated with $I_{L}=500 \mathrm{~W} / \mathrm{cm}^{2}$, the radiative temperature is $T_{r} \sim 180 \mathrm{eV}$. The radiative transport should be dominated by photons with energies of about $1200 \mathrm{eV}$, above the cutoff of $1 \mathrm{keV}$ taken here (see Fig. 15).

We found from simulations that even crude description of the radiation transport with a cutoff energy of about $10 \mathrm{keV}$ and with about ten energetic groups provides a sufficiently good description of the DAF structures. More attention need to be dedicated to the opacities model described below in Sec. IV.

\section{Temperature evolution in the plateau region}

The plasma in the plateau zone is almost in the local thermodynamic equilibrium because $T_{r} \approx T_{e}$. We observe that in the plateau region, the blackbody radiation law is verified (Fig. 16): $T_{r} \sim I_{L}^{1 / 4}$. This confirms that the plateau region is optically thick and explains why the radiative temperature $T_{r}$ does not vary in time if the laser intensity is constant (Fig. 17).

This conclusion applies to moderate $Z$ materials such as $\mathrm{SiO}_{2}, \mathrm{Al}$, and $\mathrm{CHBr}$. The charge state of plasma in the plateau region depends on the temperature according to the TF ionization model, $Z \sim T_{e}^{1 / 2}$, while the corona is fully ionized.

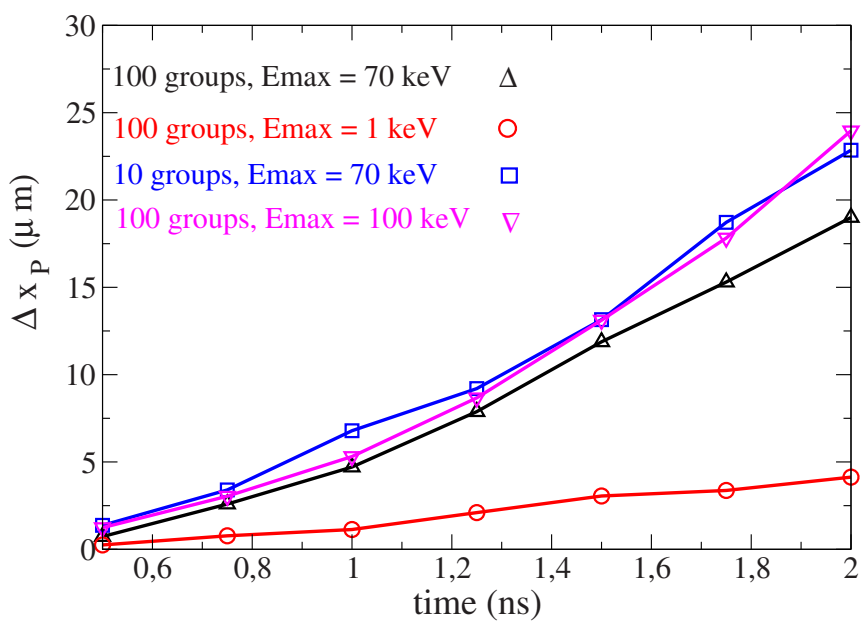

FIG. 15. (Color online) Influence of the radiation transport description on $\Delta x_{P}$ for the $\mathrm{SiO}_{2}$ target at $I_{L}=500 \mathrm{TW} / \mathrm{cm}^{2}$. 


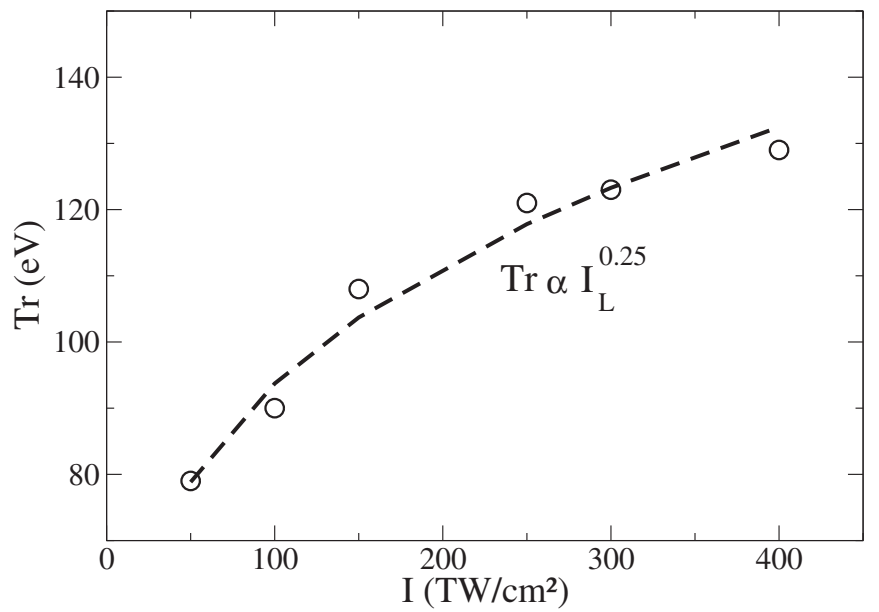

FIG. 16. Dependence of the radiative temperature $T_{r}$ in the plateau region on the laser intensity for the Al target during the acceleration stage.

\section{Density profiles in the ablation fronts}

The density profile can be characterized by two points $\rho_{\max }$ and $\rho_{P}$, as it is shown in Fig. 18. There, $\rho_{\max }$ corresponds to maximum density at the main ablation front and $\rho_{P}$ is the plateau density $\left(\rho_{\max }>\rho_{P}\right)$.

We found an empirical relation between these two parameters,

$$
\rho_{P}=a I_{L}+b \rho_{\max },
$$

with $I_{L}$ in $\mathrm{TW} / \mathrm{cm}^{2}$ and $\rho$ in $\mathrm{g} \mathrm{cm}^{-3}$, with $a$ and $b$ being two constant values. For aluminum, $a=0.0009\left(\mathrm{~g} \mathrm{~cm}^{-3}\right) /$ (TW cm $\mathrm{cm}^{-2}$ ) and $b=0.1$. The dependence (2) is shown in Fig. 19. For longer times, when the rarefaction wave arrives at the ablation front, the maximum density is lower than the initial target density and this relation is no more valid.

\section{Mass ablation rate in the DAF}

The radiative transport affects the ablation scaling laws. ${ }^{5}$ For the $\mathrm{CH}$ target, where the electron transport dominates, the ablated mass rate scales as $\dot{m}_{a} \propto I_{L}^{1 / 3}$. For the $\mathrm{CHBr}$ target,

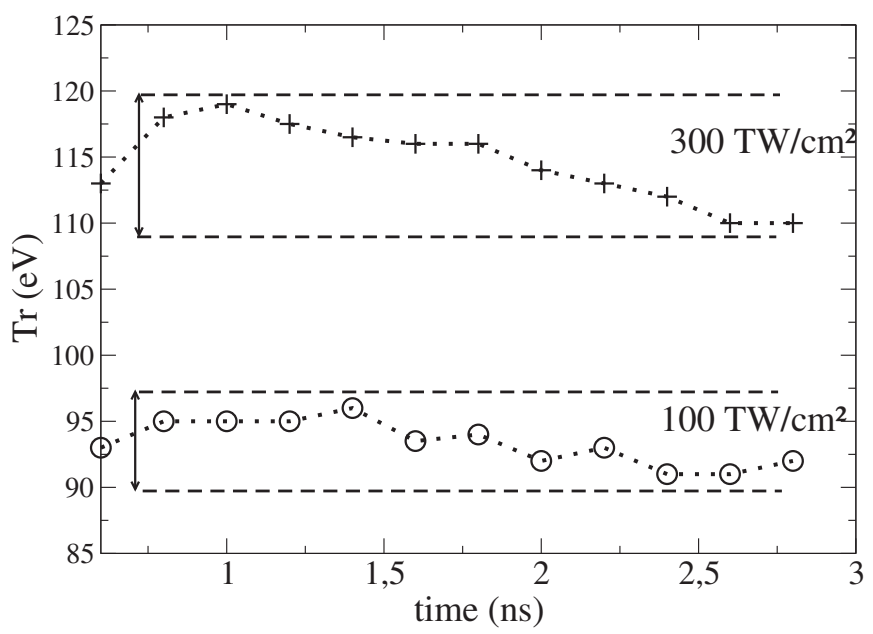

FIG. 17. Evolution of $T_{r}$ in the plateau region with time for the $\mathrm{Al}$ target during the acceleration stage.

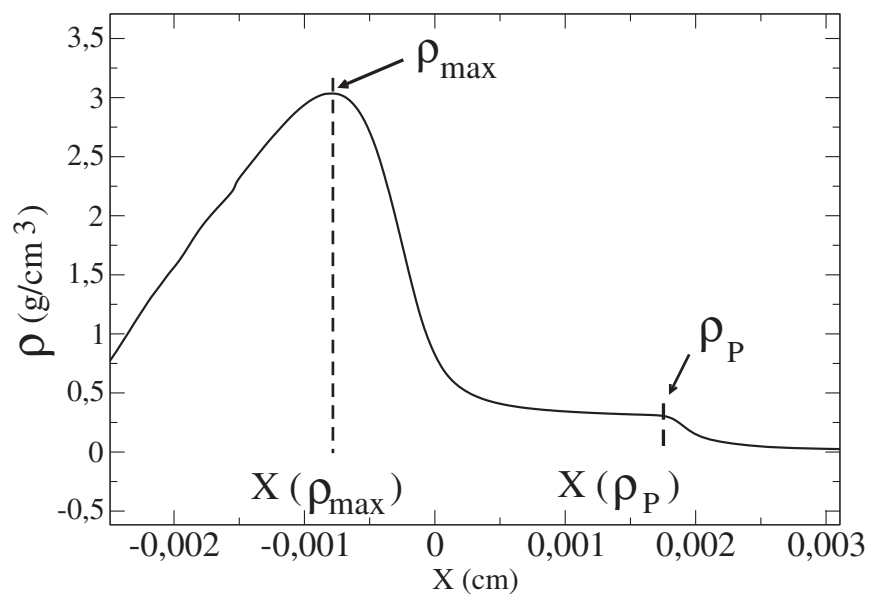

FIG. 18. Localization of $\rho_{\max }$ and $\rho_{P}$ on DAF structure density profile.

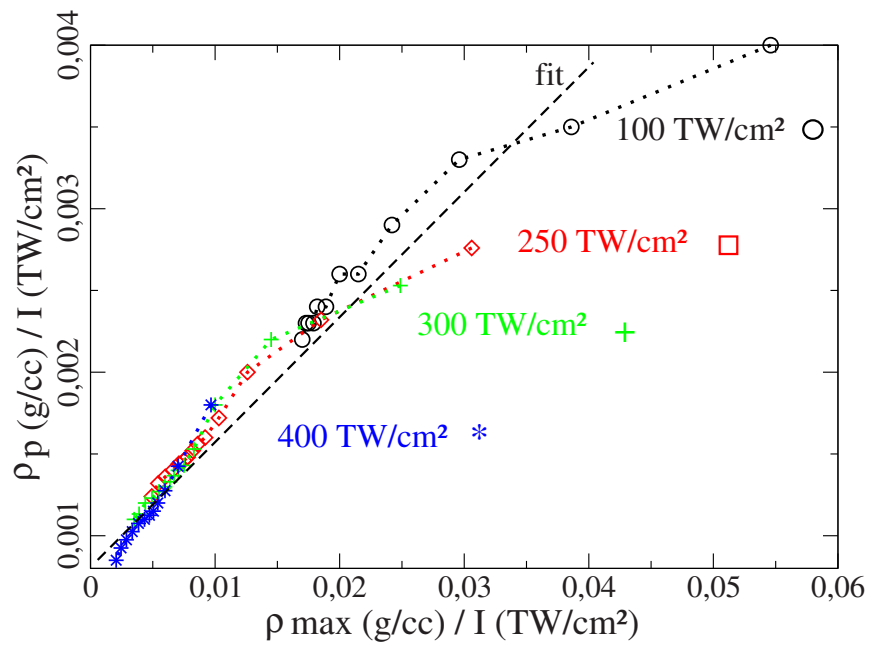

FIG. 19. (Color online) Evolution of $\rho_{P} / I_{L}$ vs $\rho_{\max } / I_{L}$ for different laser intensities laser at time $1.2 \mathrm{~ns}$ for the $\mathrm{Al}$ target.

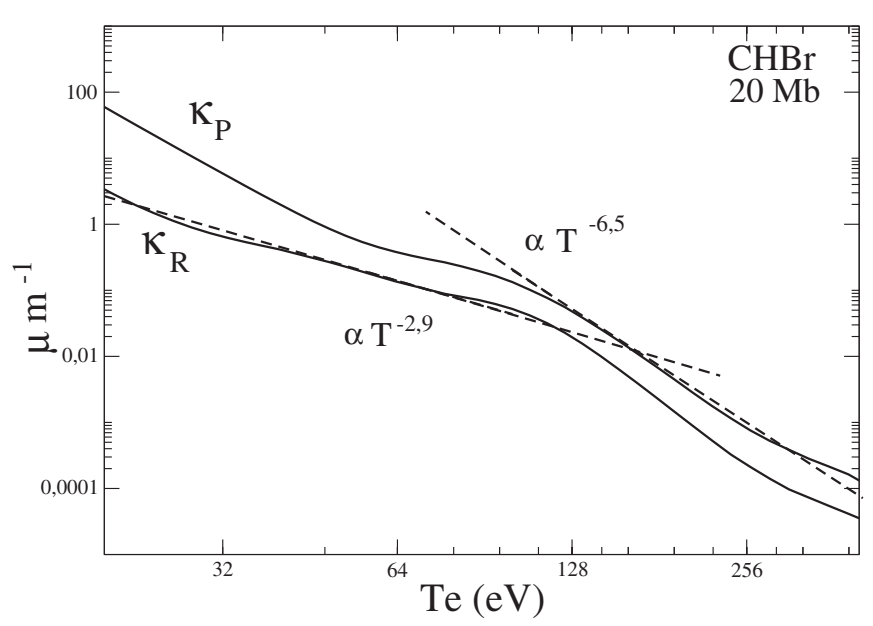

FIG. 20. Mean Rosseland and Planck opacities $\left(\mu \mathrm{m}^{-1}\right)$ for brominated plastic $\mathrm{CHBr}$ vs electron temperature $T_{e}(\mathrm{eV})$ for the pressure of $20 \mathrm{Mbar}$. 
we found that the $\dot{m}_{a}$ decreases with time $\dot{m}_{a} \propto t^{-0.22}$, but it depends strongly on the laser intensity as $I_{L}^{0.55}$. For ablation pressure $P_{a}$, we found the dependence $P_{a} \propto I_{R}^{5 / 6}$. It is found that for the CHBr target, $\dot{m}_{a}$ is higher than for the $\mathrm{CH}$ one. For example, at the time $t=1.25 \mathrm{~ns}$, the calculated values are $\dot{m}_{a} \sim 5.1 \times 10^{5} \mathrm{~g} /\left(\mathrm{cm}^{2} \mathrm{~s}\right)$ for the $\mathrm{CHBr}$ target and $\sim 2.9 \times 10^{5} \mathrm{~g} /\left(\mathrm{cm}^{2} \mathrm{~s}\right)$ for the $\mathrm{CH}$ one.

They are in the same range as calculated in Ref. 18. The higher value of the mass ablation rate corresponds to a higher value of the ablation velocity; that may have a stabilizing effect on the ablation front.

\section{COMPARISON WITH THE THEORETICAL MODEL FOR DAF STRUCTURES}

A theoretical model describing the physical processes that occurred in the DAF structures was developed in Ref. 16. There, the Kull's analytic theory of isobaric and subsonic steady ablation front with the ideal gas equation of state ${ }^{29-32}$ was extended to include both the electron and radiation energy fluxes. The electron heat flux $\vec{Q}_{e}$ was modeled with Spitzer conductivity $-\kappa_{e} T^{\nu}$. A diffusion gray approximation was used for the radiation flux $S_{r}$, according to the equations

$$
\begin{aligned}
& \operatorname{div} \vec{S}_{r}=4 \sigma_{B} \kappa_{P}\left(T_{e}^{4}-T_{r}^{4}\right), \\
& \vec{S}_{r}=-\frac{4 \sigma_{B}}{3 \kappa_{R}} \vec{\nabla} T_{r}^{4},
\end{aligned}
$$

where $\kappa_{R}$ and $\kappa_{P}$ are the Rosseland and Planck mean opacities, respectively. These are described usually by power dependencies $^{2,33}$ on the density and electron temperature

$$
\kappa_{P}=r \kappa_{R}=A T_{e}^{q_{a}} \rho^{q_{b}},
$$

assuming that their ratio $r$ is constant.

However, the domains of application of such expressions are restricted because the change of the ionization level implies the change of the value of power factors $q_{a}$ and $q_{b}$ (Fig. 20). Such simplified expressions for opacities are insufficient for DAF structure description. They need to be improved by taking into account a change of slope for a given transition temperature $T_{t}$, which depends only on the target material. ${ }^{16}$ The opacities are then described by the following expression:

$$
\begin{aligned}
& \kappa_{R}=A_{1} T_{e}^{q_{a 1}} \rho^{q_{b 1}} \quad \text { if } T_{e}<T_{t} \\
& =A_{2} T_{e}^{q_{a 2}} \rho^{q_{b 2}} \text { if } T_{e}>T_{t} .
\end{aligned}
$$

The validity of such a theoretical model was studied by comparison with the hydrodynamic profiles obtained with the CHIC code.

By choosing appropriate parameters of the model, one can obtain a pretty good agreement with the full radiation hydrodynamic simulations (see Figs. 21 and 22). Table I provides, for the $\mathrm{SiO}_{2}$ target, the values of the temperature in the main ablation front $T_{a}$, the density of the main ablation front $\rho_{a}$ and of the plateau region $\rho_{f}$, and the length of the plateau $\Delta x_{P}$. In spite of its simplicity, the theoretical model compares
TABLE I. Parameters for the opacities modelization for $\mathrm{CH}, \mathrm{CHBr}$, and $\mathrm{SiO}_{2}$ targets.

\begin{tabular}{lcc}
\hline \hline & Theoretical model & CHIC code \\
\hline$T_{a}(\mathrm{eV})$ & 46 & 35 \\
$\rho_{a}\left(\mathrm{~g} / \mathrm{cm}^{3}\right)$ & 2.0 & 1.9 \\
$\rho_{f}\left(\mathrm{~g} / \mathrm{cm}^{3}\right)$ & 0.72 & 0.68 \\
$\Delta x_{P}(\mu \mathrm{m})$ & 8 & 12 \\
\hline \hline
\end{tabular}

well thefull simulations. The main parameters of the radiation transport model are shown in Table II. For Al, the opacity approximation with one $T_{t}$ is not sufficient. We approximated it with two temperatures $T_{t 1}$ and $T_{t 2}$, assuming a constant $\kappa_{r}$ and $\kappa_{P}$ between them.

According to the model, ${ }^{16}$ the condition of formation of the second ablation front is defined by the value of the parameter $\beta_{t}$,

$$
\beta_{t}=\frac{16}{25} \frac{\left(\sigma_{B} T_{t}^{4} \kappa_{P t}\right)\left(\kappa_{e t} T_{t}\right)}{\left(P_{t} v_{t}\right)^{2}}>1,
$$

where the subscript $t$ refers to the conditions at the second ablation front, $\kappa_{e}$ is the coefficient of electron heat conductivity, $v_{t}$ is the electron thermal velocity, and $P$ is the pressure.

Indeed, in our CHIC simulations we found the following values for $\beta_{t}$ :

(a) for $\mathrm{CH}, \beta_{t} \sim 1$, no DAF;

(b) for $\mathrm{CHBr}, \beta_{t} \sim 6.4$, DAF is formed; and

(c) for $\mathrm{SiO}_{2}, \beta_{t} \sim 19.4$, DAF is very well defined.

This simplified model with an ideal gas equation of state and power law opacities [Eq. (4)] is then used to introduce the radiative transport into the PERLE code in order to study the stability of DAF structures.

\section{STABILITY OF DAF STRUCTURES}

PERLE (Refs. 25 and 26) is a code dedicated to studies of temporal and spatial evolution of 2D/3D linear perturbations of 1D unsteady flows. This code considers a plasma as an ideal gas, the laser energy release is modeled by the inverse bremsstrahlung absorption; the electron thermal transport is described in a Spitzer-Harm approximation. The radiative transport is introduced in the diffusion approximation with a gray model for the mean Planck and Rosseland opacities, according to Eqs. (3) and (4). The appropriate parameters are

TABLE II. Comparison of the DAF parameters obtained with a simplified theoretical model and full radiation hydrodynamic calculation for $\mathrm{CH}$, $\mathrm{CHBr}, \mathrm{SiO}_{2}$, and $\mathrm{Al}$ targets.

\begin{tabular}{lcccccc}
\hline \hline & & & $T_{t}$ & $T_{t 1}$ & $T_{t 2}$ & \\
& $q_{1}$ & $q_{2}$ & $(\mathrm{eV})$ & $(\mathrm{eV})$ & $(\mathrm{eV})$ & $r$ \\
\hline $\mathrm{CH}$ & -2.1 & -5.7 & 90 & $\ldots$ & $\ldots$ & 6 \\
$\mathrm{CHBr}_{4 \%}$ & -1.9 & -4.5 & 130 & $\ldots$ & $\ldots$ & 3 \\
$\mathrm{SiO}_{2}$ & -2 & -4.2 & 160 & $\ldots$ & $\ldots$ & 3 \\
$\mathrm{Al}$ & -3 & -4.5 & $\cdots$ & 200 & 315 & 3 \\
\hline \hline
\end{tabular}



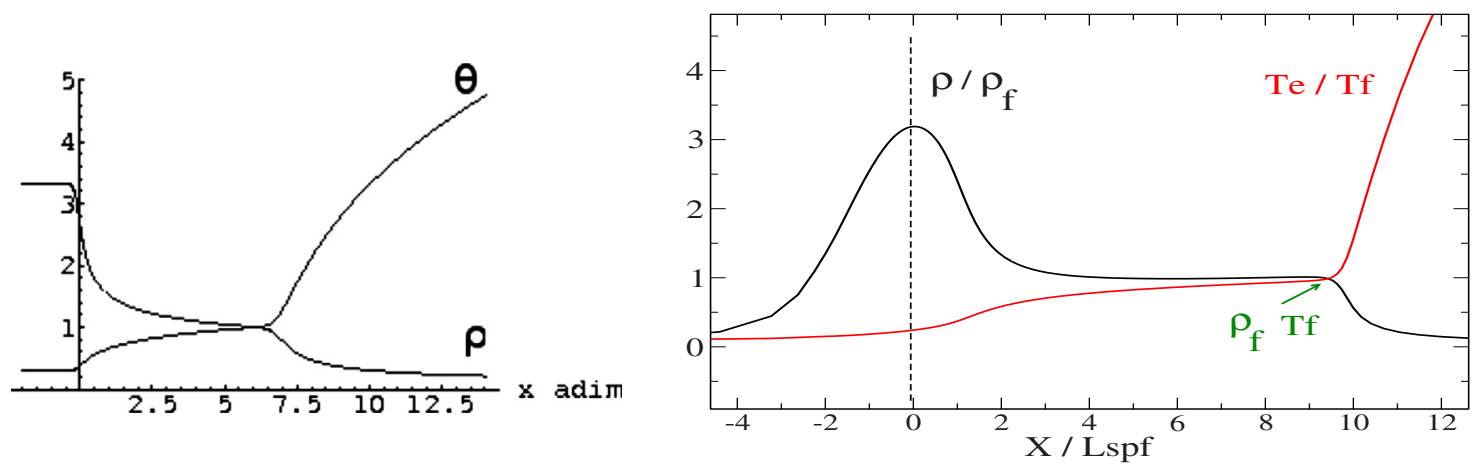

FIG. 21. (Color online) Profiles of density $\rho$ and electronic temperature $\theta$ normalized with respective values at the second ablation front for $\mathrm{SiO}_{2}$ target during the acceleration stage $(t \sim 1.3 \mathrm{~ns})$ at laser intensity of $I_{L}=500 \mathrm{TW} / \mathrm{cm}^{2}$, obtained by solving the theoretical model equations (left) and with the CHIC code (right).
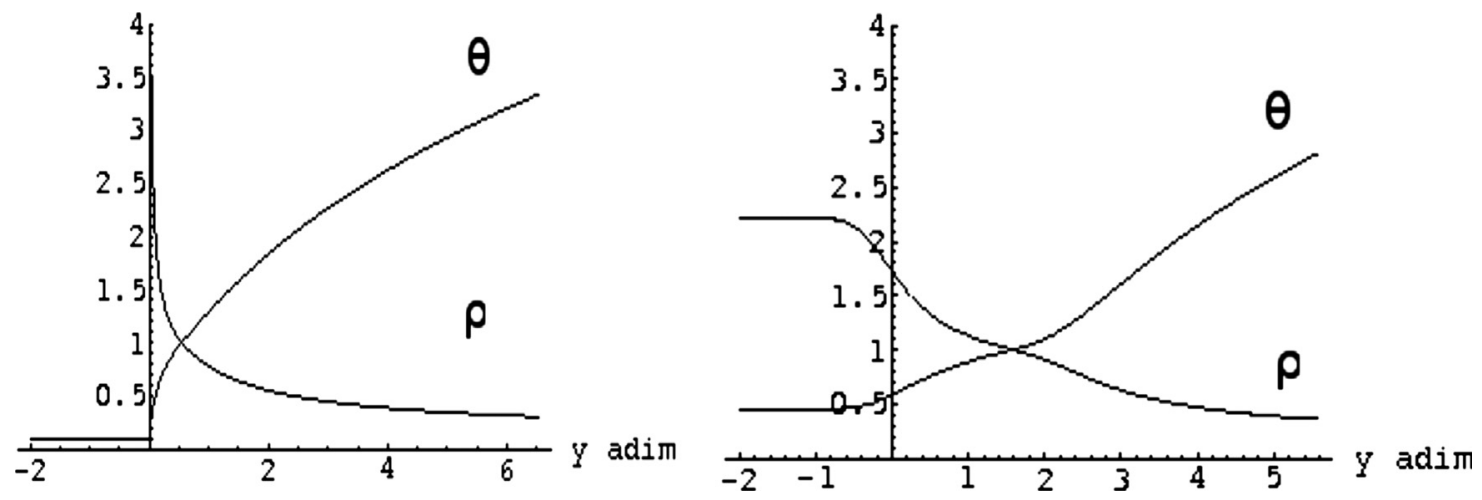

FIG. 22. Profiles of density $\rho$ and electronic temperature $\theta$ normalized with respective values at the second ablation front for $\mathrm{CH}$ (left: classical ablation structure) and $\mathrm{CHBr}$ (right: little DAF structure) targets during the acceleration stage $(t \sim 1.1 \mathrm{~ns})$ at laser intensity of $I_{L}=200 \mathrm{TW} / \mathrm{cm}^{2}$, obtained by solving the theoretical model equations.
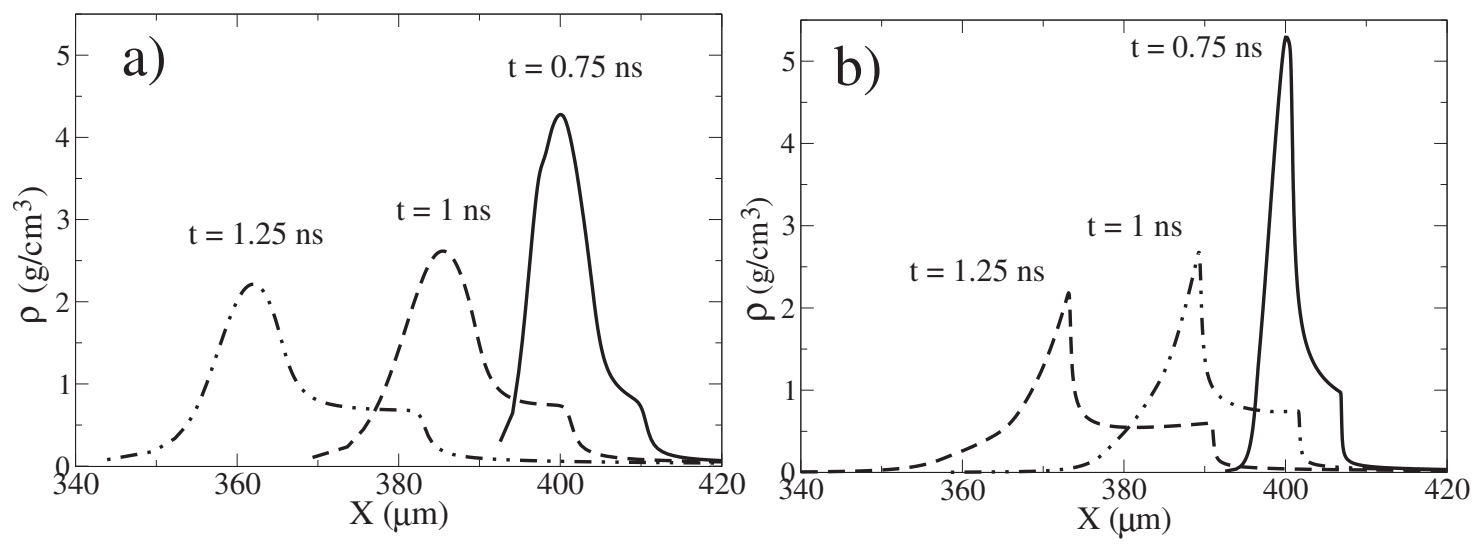

FIG. 23. Evolution of density profiles obtained with the CHIC (a) and PERLE (b) codes for $\mathrm{SiO}_{2}$ target during the acceleration stage for $I_{L}=100 \mathrm{TW} / \mathrm{cm}^{2}$. 


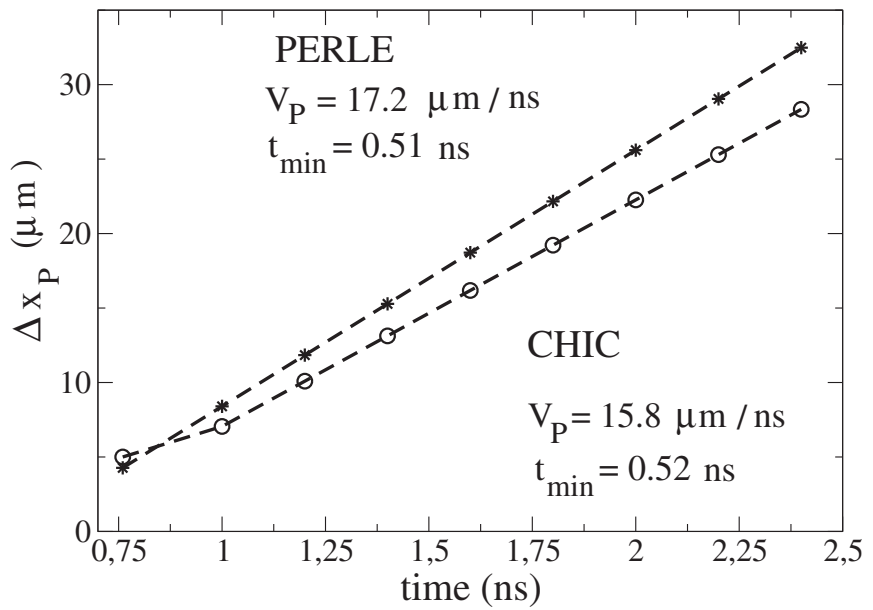

FIG. 24. Evolution of the plateau length $\Delta x_{P}$ in time calculated with the PERLE and CHIC codes for Al target and $I_{L}=100 \mathrm{TW} / \mathrm{cm}^{2}$.

given in Table II. The evolution of perturbations of DAF structures calculated with PERLE is compared with full 2D simulations using CHIC.

\section{A. DAF structures obtained with PERLE}

In spite of the fact that the PERLE physical model is simpler than that of the CHIC, very similar structures are obtained by choosing appropriate parameters for the opacities. With PERLE, the characteristic thickness of the main ablation front $L_{0}$ is only slightly underestimated, and the chronology is respected. Besides, we found the same evolution of wave structures with the time and laser intensity, and the plateau width increases in time with approximately the same velocity (see Fig. 23). We found, for example, for the silica target from PERLE calculation $V_{P}=17.2 \mu \mathrm{m} / \mathrm{ns}$ and $t_{\min }=0.51 \mathrm{~ns}$ (Fig. 24)

The DAF structures have been obtained with PERLE in other materials ( $\mathrm{Al}, \mathrm{CH}$, and $\mathrm{CHBr}_{4 \%}$ ), and we found again a good agreement with CHIC. The differences are mainly due to the modeling of the radiative transfer. It was verified by conducting simulations with the CHIC code with a very few energy groups. The quantitative comparison of the CHIC and PERLE results is presented in Table III for the ablation velocity $V_{a}$, acceleration $g$, the first ablation front thickness $L_{0}$, and the Froude number $\mathrm{Fr}=V_{a}^{2} / g L_{0}$ for three materials. The minimum density gradient length $L_{m}$ was estimated too. This parameter is used in Eq. (6) and in the isobaric model ${ }^{31,32}$ to

TABLE III. Comparison of ablation characteristic parameters obtained with the CHIC and PERLE codes for $\mathrm{CH}, \mathrm{CHBr}$, and $\mathrm{Al}$.

\begin{tabular}{|c|c|c|c|c|c|c|}
\hline & \multicolumn{2}{|c|}{$\mathrm{CH}$} & \multicolumn{2}{|c|}{$\mathrm{CHBr}_{4 \%}$} & \multicolumn{2}{|c|}{$\mathrm{Al}$} \\
\hline & PERLE & CHIC & PERLE & CHIC & PERLE & CHIC \\
\hline$V_{a}(\mu \mathrm{m} / \mathrm{ns})$ & 1.8 & 2 & 3.5 & 3.7 & 1.9 & 2.1 \\
\hline$g\left(\mu \mathrm{m} / \mathrm{ns}^{2}\right)$ & 150 & 144 & 90 & 84 & 25.3 & 24.1 \\
\hline$L_{0}(\mu \mathrm{m})$ & 0.13 & 0.2 & 1.8 & 1 & 2 & 0.98 \\
\hline $\mathrm{Fr}$ & 0.16 & 0.12 & 0.13 & 0.1 & 0.16 & 0.1 \\
\hline$T_{r}(\mathrm{eV})$ & $\cdots$ & $\cdots$ & 88 & 110 & 85 & 100 \\
\hline
\end{tabular}

estimate the Rayleigh-Taylor instability growth rate of classical ablation front structures

$$
L_{0}=L_{m} \frac{\nu^{\nu}}{(\nu+1)^{\nu+1}},
$$

where $\nu=5 / 2$ is the power temperature dependence of the electron heat conductivity.

In the first case, we consider a $20 \mu \mathrm{m}$ thick $\mathrm{CH}$ target, with $I_{L}=200 \mathrm{TW} / \mathrm{cm}^{2}$ at the time $t=1.3 \mathrm{~ns}$. In the second case, we consider a $20 \mu \mathrm{m}$ thick $\mathrm{CHBr}_{4 \%}$ target, with $I_{L}$ $=200 \mathrm{TW} / \mathrm{cm}^{2}$ at the same time $t=1.3 \mathrm{~ns}$. In the third case, we consider a $20 \mu \mathrm{m}$ thick Al target, with $I_{L}$ $=100 \mathrm{TW} / \mathrm{cm}^{2}$ at the time $t=1.5 \mathrm{~ns}$. All cases correspond to the acceleration stage.

For the $\mathrm{CH}$, the front is thinner in the PERLE calculation, but the flow characteristics are very similar. The same conclusions apply to other materials.

\section{B. $\mathrm{RTI}$ growth rate in $\mathrm{CHBr}$ and $\mathrm{CH}$ targets}

The parameters of the ablation fronts calculated with CHIC and PERLE codes allow one to evaluate the stability of these fronts. With the PERLE code, the RTI growth rate is calculated for several perturbation wave numbers and different times by estimating the slope of a single wave number perturbation amplitude evolution in time at the ablation front.

First, we studied the stability of the main ablation front in the $\mathrm{CH}$ and $\mathrm{CHBr}$ targets during the acceleration stage. The laser intensity has a linear ramp of $100 \mathrm{ps,} \mathrm{followed} \mathrm{by}$ a constant intensity $I_{L}=200 \mathrm{TW} / \mathrm{cm}^{2}$. The same laser profile was used in Refs. 31 and 32, so the results can be compared. Betti et al. $^{31,32}$ assumed an isobaric approximation near a single ablation front region, and the energy transport was modeled with a single temperature $\vec{Q}_{e}=-\kappa_{e} T^{\nu} \vec{\nabla} T$. Then the parameters for the energy flux $\kappa_{e}$ and $\nu$ were modified to take into account the radiative transport in a simplified way. The temperature and density profiles obtained in the simulations were compared with the profiles found from the equations of the isobaric model. ${ }^{31}$ Then the flow parameters were estimated, and the growth rate was calculated. On the contrary, in our simulations with PERLE, a DAF structure can be formed because two energy transport mechanisms were considered explicitly.

The dispersion curve for the $\mathrm{CH}$ target corresponds to the classical ablation front. It can be fitted with the modified Takabe-like ${ }^{31,34}$ formula

$$
\gamma \approx \alpha \sqrt{\frac{k g}{1+k L_{m}}}-\beta k V_{a} .
$$

The coefficients $\alpha$ and $\beta$ are calculated using self-consistent formulas derived in Refs. 31 and 32 and are dependent on the Froude number, the material density jump, and the ablation velocity.

In this case, the coefficients $\alpha=0.91$ and $\beta=1.66$ are in agreement with values given in Refs. 31 and 32 for the front thickness of $0.7 \mu \mathrm{m}<L_{m}<1 \mu \mathrm{m}$. Here, we estimate $L_{m}$ $\sim 0.9 \mu \mathrm{m}$.

The RTI growth rate in the $\mathrm{CHBr}$ target is reduced in comparison to the $\mathrm{CH}$. The cutoff is displaced toward 


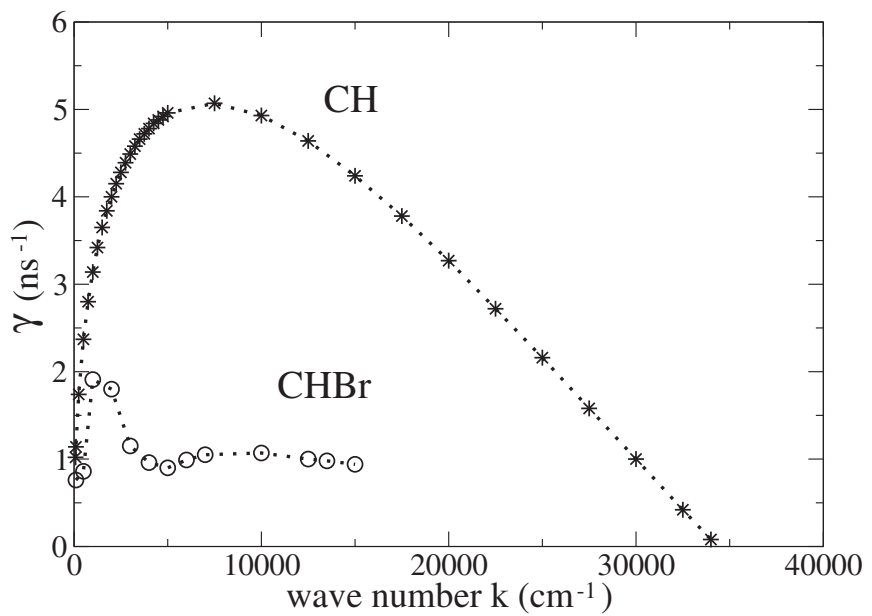

FIG. 25. Growth rates $\gamma$ of the RT instability calculated for $\mathrm{CH}(+)$ and $\mathrm{CHBr}(\bigcirc)$ with PERLE at the time of $1.5 \mathrm{~ns}$.

smaller wave numbers. A new set of unstable wave numbers appears for higher wave numbers. The reduction of the maximum growth rate is partially due to a smaller acceleration (see Table III), but the modification in the dispersion curve shape is associated with the second ablation front influence (see Fig. 25).

\section{RTI growth rate for $\mathrm{Al}$ and $\mathrm{SiO}_{2}$ targets}

The RTI growth rates calculated for $\mathrm{Al}$ and $\mathrm{SiO}_{2}$ targets irradiated by $I_{L}=100 \mathrm{TW} / \mathrm{cm}^{2}$ laser light, using PERLE, are shown in Fig. 26. Similar to the case of $\mathrm{CHBr}$, the dispersion curve consists of two lobes separated by a narrow dip, where the perturbations are stabilized. The second lobe at higher wave numbers is more pronounced than for the $\mathrm{CHBr}$ and the maximum values are of the same order in the both lobes. This may be owed to the fact that the DAF structure is better defined in these materials due to enhanced radiative effects.

For the $\mathrm{SiO}_{2}$ target, the second lobe appears for the modes with the wave numbers higher than $k_{d} \sim 9200 \mathrm{~cm}^{-1}$. In general, we observe that the wavelength corresponding to the dip correlates with the plateau length. As an example, in $\mathrm{SiO}_{2}$ at $t \sim 1.5 \mathrm{~ns}, \lambda_{d} \sim 7 \mu \mathrm{m}$, and $\Delta x_{P} \sim 13 \mu \mathrm{m}$. In $\mathrm{Al}$, $\lambda_{d} \sim 8 \mu \mathrm{m}$ at $t \sim 1.5 \mathrm{~ns}$, while the length of the plateau is $\Delta x_{P} \sim 14 \mu \mathrm{m}$.

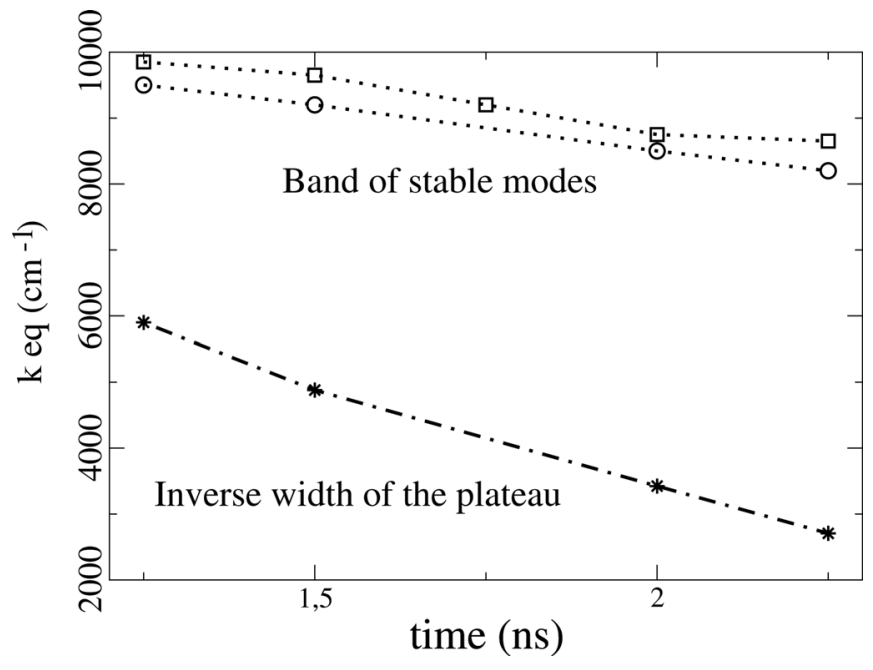

FIG. 27. Evolution of the band of stable modes and the inverse plateau length $2 \pi / \Delta x_{P}$ with time for the $\mathrm{SiO}_{2}$ target.

When calculating the growth rate evolution with time in the interval between 1.25 and $2.25 \mathrm{~ns}$, we found that the wavelength of the dip increases with the plateau size (Fig. 27). We found that the short wavelength modes, smaller than the plateau characteristic length develop locally at each front, without notable influence between them. We found that they are localized near from the main front. The perturbations with intermediate wavelengths of the order of the plateau length are stabilized probably due to coupling of the perturbations at both fronts. Correspondingly, a dip in the dispersion curve appears. The perturbations with the larger wavelengths do not experience stabilizing effects of the plateau and of the second front. They are localized near the main front and a single front RTI model is probably appropriate for their description. Our simulations demonstrate a clear correlation between the dispersion curve shape and the DAF structure. However, the influence of the nonstationary and convective effects may also have an influence, but it was not investigated here.

\section{Estimates of RTI growth rates with CHIC}

Two-dimensional simulations with the CHIC code have also been performed to study the evolution of the ablation front perturbation amplitudes. In spite of a much longer com-
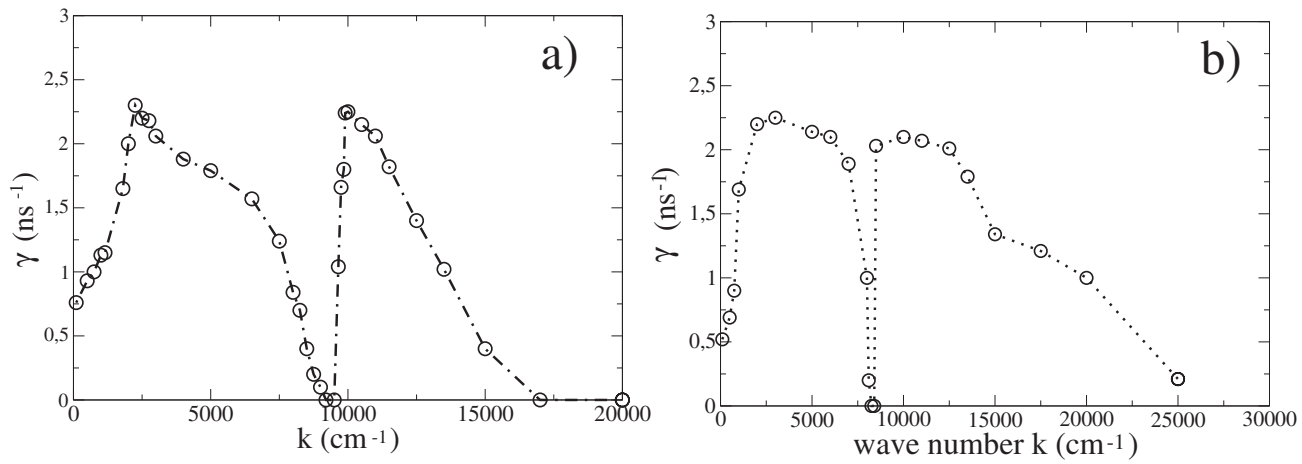

FIG. 26. RTI Growth rates calculated for $\mathrm{SiO}_{2}$ (a) and Al (b) with PERLE (O) at time $t=1.5 \mathrm{~ns}$ and $I_{L}=100 \mathrm{TW} / \mathrm{cm}^{2}$. 


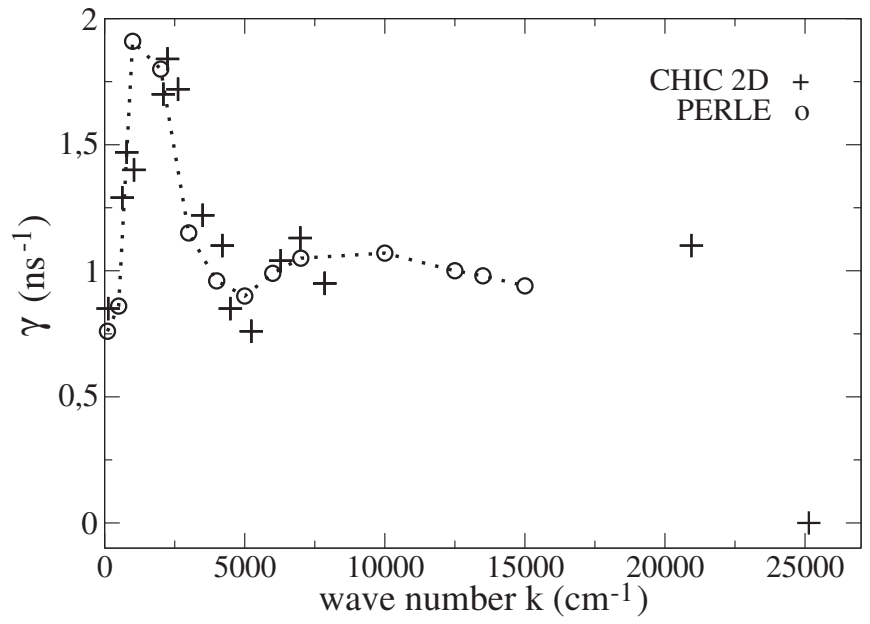

FIG. 28. Growth rate of the ablation front instability obtained with the codes CHIC 2D (+) and PERLE (O) for the CHBr target at $1.5 \mathrm{~ns}$.

putational time, this code includes a realistic and complete physics. Thus, the growth rate calculation can be performed in a more complete manner. However, the perturbations associated with the very small wavelengths (few micrometers) are difficult to calculate: strong distortion of the mesh in the perpendicular direction requires mesh refinement and reduces precision.

To estimate the growth rate of a given perturbation, CHIC code simulations are run with periodic boundary conditions in the perpendicular direction, and we calculated the "peak to valley" amplitude during the simulation. An initial periodic perturbation with the wavelength twice the simulation box length is considered.

Acquired results confirm the growth rate values and the presence of a dip in the dispersion curve for the $\mathrm{CHBr}$ targets (Fig. 28). Values of growth rate calculated with CHIC 2D are in a very good agreement with values calculated with PERLE.

Growth rates have also been calculated with CHIC for the $\mathrm{CH}$ and $\mathrm{SiO}_{2}$ targets (Fig. 29). For a $\mathrm{SiO}_{2}$ target, calculations for wave numbers higher than $10000 \mathrm{~cm}^{-1}$ are not yet achieved with CHIC 2D because of insufficient resolution in the perpendicular direction.

\section{CONCLUSIONS}

We have investigated ablation structures in moderate $Z$ and doped materials. The structures containing two ablation fronts separated by a constant density region are found. They are due to a competition between the electron and the radiative transport mechanisms.

Radiation hydrodynamic simulations with the CHIC code have permitted to characterize quantitatively the DAF structures in the range of laser intensities $1-500 \mathrm{TW} / \mathrm{cm}^{2}$ for the silica, aluminum, and bromine doped plastic targets. The influence of ionization and equation of state models has been investigated and it is confirmed that the radiation transport is the dominant effect for the DAF dynamics.

A simplified model of radiation transport ${ }^{16}$ with a piecewise interpolation of opacities is shown to be relevant for the description of DAF structures. By comparing the CHIC and PERLE simulations, we found very similar hydrodynamic profiles and characteristic parameters of the ablation structure. Simple empirical formulas for the DAF structure parameters are deduced from simulations.

The stability of DAF structures was studied. Unlike the instability of a single front, the dispersion curve consists of two lobes separated by a narrow dip where the perturbations are stabilized. The maximum growth rate is reduced as well as the cutoff wave number. These modifications may be due to the combined effects of the double ablation front and a strong nonstationarity of the flow.

A good agreement between the growth rates obtained with the CHIC and PERLE codes confirms the relevance of simplified models for both the 1D evolution and the stability study of the DAF. The use of moderate $Z$ ablators could be a promising way to control the stability of ablation front in ICF targets.

\section{ACKNOWLEDGMENTS}

This work was supported in part by the Aquitaine Region council. This work was also supported by the Ministerio de Ciencia y Tecnologia of Spain (Grant No. FTN200762485). a)

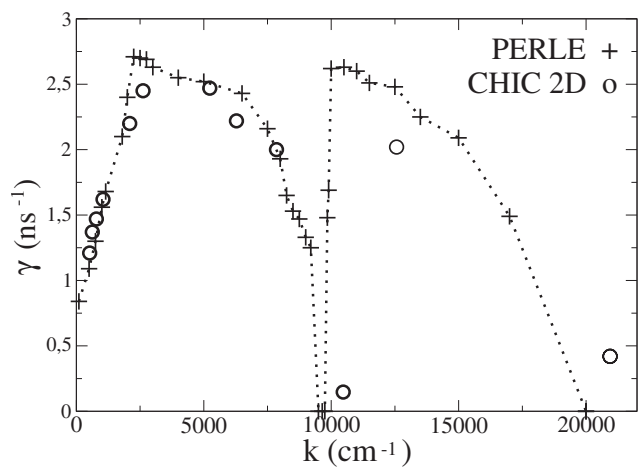

b)

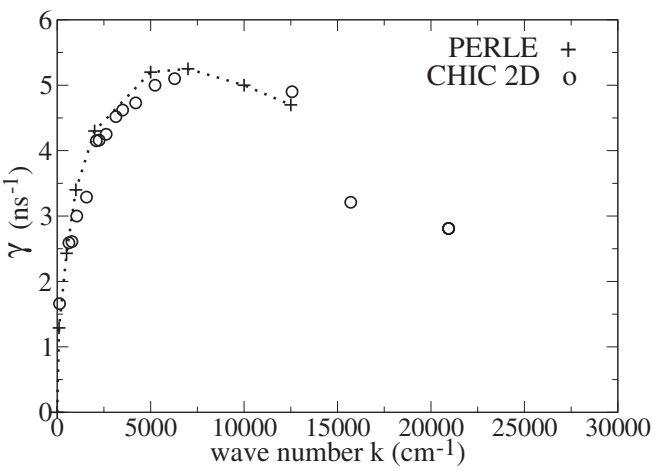

FIG. 29. Growth rates of the ablation front instability calculated with CHIC 2D (O) and PERLE $(+)$ for the $\mathrm{SiO}{ }_{2}$ target at 1.25 ns $\left(\right.$ a) and the $\mathrm{CH}_{\text {target }}$ at $1.5 \mathrm{~ns}(\mathrm{~b})$. 
${ }^{1}$ J. D. Lindl, Phys. Plasmas 2, 3933 (1995).

${ }^{2}$ R. P. Drake, High Energy Density Physics (Springer, New York, 2006).

${ }^{3}$ D. Mihalas and B. W. Mihalas, Foundation of Radiation Hydrodynamics (Dover, New York, 2000).

${ }^{4}$ R. E. Marshak, Phys. Fluids 1, 24 (1958).

${ }^{5} \mathrm{R}$. Dautray and J. P. Watteau, La fusion thermonucléaire inertielle par laser, Collection du CEA (Eyrolles, Paris, 1993).

${ }^{6}$ R. Pakula and R. Sigel, Phys. Fluids 28, 232 (1985).

${ }^{7}$ Y. B. Zel'dovich and Y. P. Raizer, Physics of Shock Waves and High Temperature Hydrodynamic Phenomena (Academic, New York, 1967).

${ }^{8}$ W. M. Manheimer, D. G. Colombant, and J. H. Gardner, Phys. Fluids 25, 1644 (1982).

${ }^{9}$ N. Kaiser, J. Meyer-Ter-Vehn, and R. Sigel, Phys. Fluids B 1, 1747 (1989).

${ }^{10}$ R. F. Schmalz, J. Meyer-ter-Vehn, and R. Ramis, Phys. Rev. A 34, 2177 (1986).

${ }^{11}$ D. Duston, R. W. Clark, J. Davis, and J. P. Apruzese, Phys. Rev. A 27, 1441 (1983).

${ }^{12}$ R. Sigel, K. Eidmann, F. Lavarenne, and R. F. Schmalz, Phys. Fluids B 2, 199 (1990).

${ }^{13}$ K. Eidmann, R. F. Schmalz, and R. Siegel, Phys. Fluids B 2, 208 (1990).

${ }^{14}$ R. Sigel, G. D. Tsakiris, F. Lavarenne, J. Massen, R. Fedosejevs, J. Meyerter-Vehn, M. Murakami, K. Eidmann, S. Witkowski, H. Nishimura, Y. Kato, H. Takabe, T. Endo, K. Kondo, H. Shiraga, S. Sakabe, T. Jitsuno, M. Takagi, C. Yamanaka, and S. Nakai, Phys. Rev. Lett. 65, 587 (1990).

${ }^{15}$ V. A. Smalyuk, S. X. Hu, V. N. Goncharov, D. D. Meyerhofer, D. C. Sangster, D. Shvarts, C. Stoeckl, and B. Yaakobi, Phys. Rev. Lett. 101, 025002 (2008).

${ }^{16}$ J. Sanz, R. Betti, V. A. Smalyuk, M. Olazabal-Loumé, V. Drean, V. T. Tikhonchuk, X. Ribeyre, and J. L. Feugeas, Phys. Plasmas 16, 082704 (2009).

${ }^{17}$ V. A. Smalyuk, R. Betti, J. A. Delettrez, V. Yu. Glebov, D. D. Meyerhofer, P. B. Radha, S. P. Regan, T. C. Sangster, J. Sanz, W. Seka, C. Stoeckl, B.
Yaakobi, J. A. Frenje, C. K. Li, R. D. Petrasso, and F. H. Séguin, Phys. Rev. Lett. 104, 165002 (2010).

${ }^{18}$ S. Fujioka, A. Sunahara, N. Ohnishi, Y. Tamari, K. Nishihara, H. Azechi, H. Shiraga, M. Nakai, K. Shigemori, T. Sakaiya, M. Tanaka, K. Otani, K. Okuno, T. Watari, T. Yamada, M. Murakami, K. Nagai, T. Norimatsu, Y. Izawa, S. Nozaki, and Y.-W. Chen, Phys. Plasmas 11, 2814 (2004).

${ }^{19}$ P. H. Maire, J. Comput. Phys. 228, 2391 (2009).

${ }^{20}$ P. H. Maire, R. Abgrall, J. Breil, and J. Ovadia, SIAM J. Sci. Comput. (USA) 29, 1781 (2007).

${ }^{21}$ P. H. Maire, J. Breil, and S. Galera, Int. J. Numer. Methods Fluids 56, 1161 (2008)

${ }^{22}$ P. H. Maire and J. Breil, Int. J. Numer. Methods Fluids 56, 1417 (2008).

${ }^{23}$ J. Breil and P. H. Maire, J. Comput. Phys. 224, 785 (2007).

${ }^{24}$ P. Nicolaï, J. L. Feugeas, and G. Schurtz, Phys. Plasmas 13, 032701 (2006).

${ }^{25}$ E. Godlewski, M. Olazabal, and P. A. Raviart, J. Math. Pures Appl. 78, 1013 (1999).

${ }^{26}$ E. Godlewski, M. Olazabal, and P. A. Raviart, in Godunov's Methods: Theory and Applications, edited by E. F. Toro (Kluwer Academic/Plenum, New York, 2001), p. 377.

${ }^{27}$ M. Olazabal and L. Hallo, Phys. Plasmas 14, 10 (2007).

${ }^{28}$ L. Spitzer and R. Harm, Phys. Rev. 89, 977 (1953).

${ }^{29}$ H. J. Kull, Phys. Fluids B 1, 170 (1989).

${ }^{30}$ H. J. Kull and S. I. Anisimov, Phys. Fluids 29, 2067 (1986).

${ }^{31}$ R. Betti, V. N. Goncharov, R. L. McCrory, and C. P. Verdon, Phys. Plasmas 5, 1446 (1998); 3, 4665 (1996); 2, 3844 (1995).

${ }^{32}$ R. Betti, V. N. Goncharov, R. L. McCrory, P. Sorotokin, and C. P. Verdon, Phys. Plasmas 3, 2122 (1996); 3, 1402 (1996).

${ }^{33}$ G. D. Tsakiris and K. Eidmann, J. Quant. Spectrosc. Radiat. Transf. 38, 353 (1987).

${ }^{34}$ H. Takabe, K. Mima, L. Montierth, and R. L. Morse, Phys. Fluids 28, 3676 (1985). 